CRYSTALLOGRAPHIC COMMUNICATIONS

ISSN 2056-9890

Received 11 October 2021

Accepted 27 October 2021

Edited by J. Ellena, Universidade de Sâo Paulo, Brazil

Keywords: 4-[(benzylamino)carbonyl]-1methylpyridinium; molecular structure; crystal structure; Hirshfeld analysis.

CCDC references: $2118096 ; 2118095$

Supporting information: this article has supporting information at journals.iucr.org/e
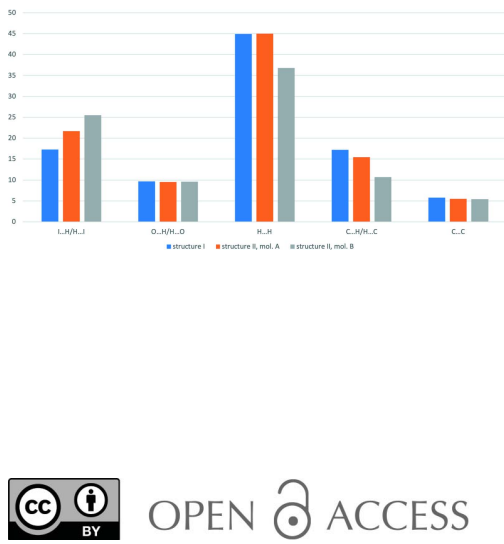

OPEN $\partial$ ACCESS

\section{Salts of 4-[(benzylamino)carbonyl]-1-methyl- pyridinium and iodide anions with different cation:iodine stoichiometric ratios}

\author{
Vitalii V. Rudiuk, ${ }^{\text {a,b }}$ Anna M. Shaposhnyk, ${ }^{c}$ Vyacheslav M. Baumer, ${ }^{\mathrm{c}}$ Igor A. \\ Levandovskiy $^{b}$ and Svitlana V. Shishkina ${ }^{\mathrm{c}, \mathrm{d}_{*}}$
}

\begin{abstract}
${ }^{\mathbf{a}}$ Farmak JSC, 63 Kyrylivska str., Kyiv 04080, Ukraine, ${ }^{\mathbf{b}}$ Department of Organic Chemistry, National Technical University of Ukraine, 37 Pobedy ave., Kyiv 03056, Ukraine, 'SSI "Institute for Single Crystals", NAS of Ukraine, 60 Nauky ave., Kharkiv 61001, Ukraine, and ' V.N. Karazin Kharkiv National University, 4 Svobody sq., Kharkiv 61022, Ukraine.

*Correspondence e-mail: sveta@xray.isc.kharkov.com
\end{abstract}

The two iodide salts, 4-[(benzylamino)carbonyl]-1-methylpyridinium iodideiodine (2/1), $\mathrm{C}_{14} \mathrm{H}_{15} \mathrm{~N}_{2} \mathrm{O}^{+} \cdot \mathrm{I}^{-} \cdot 0.5 \mathrm{I}_{2}$, I, and 4-[(benzylamino)carbonyl]-1-methylpyridinium triiodide, $\mathrm{C}_{14} \mathrm{H}_{15} \mathrm{~N}_{2} \mathrm{O}^{+} \cdot \mathrm{I}_{3}{ }^{-}$, II, with different cation:iodine atoms ratios were studied. Salt $\mathbf{I}$ contains one cation, one iodide anion and half of the neutral $\mathrm{I}_{2}$ molecule in the asymmetric unit (cation:iodine atoms ratio is $1: 2$ ). Salt II contains two cations, one triiodide anion $\left(\mathrm{I}_{\mathbf{3}}{ }^{-}\right)$and two half triiodide anions (cation:iodine atoms ratio is 1:3). The $\mathrm{NH}$ group forms $\mathrm{N}-\mathrm{H} \cdots \mathrm{I}$ hydrogen bonds with the $\mathrm{I}^{-}$anion in the crystal of $\mathbf{I}$ or $\mathrm{N}-\mathrm{H} \cdots \mathrm{O}$ hydrogen bonds in II where only triiodide anions are present.

\section{Chemical context}

4-[(Benzylamino)carbonyl]-1-methylpyridinium iodide, chemical formula $\mathrm{C}_{14} \mathrm{H}_{15} \mathrm{~N}_{2} \mathrm{O}^{+} \cdot \mathrm{I}^{-}$, is used as a multimodal antiviral drug (te Velthuis et al., 2020; Boltz et al., 2018; Buhtiarova et al., 2003; Frolov et al., 2004). Its molecular and crystal structure have been studied in detail by diffraction and spectroscopic methods (Drebushchak et al., 2017). The formation of different polymorphic modifications of an API is of great importance for the pharmaceutical industry (Bernstein, 2002; Brittain, 2009; Hilfiker, 2006). Unfortunately, all attempts to find polymorphic modifications of 4-[(benzylamino)carbonyl]-1-methylpyridinium iodide resulting from varying the solvents and crystallization conditions have failed. Only one crystal form with the $P 2_{1} 2_{1} 2_{1}$ orthorhombic space group has been determined by single-crystal X-ray diffraction (Drebushchak et al., 2017).<smiles>C[n+]1ccc(C(=O)NCc2ccccc2)cc1</smiles><smiles>C[n+]1ccc(C(=O)NCc2ccccc2)cc1</smiles>

In a continuation of this work, we attempted to obtain a new polymorphic form of this compound using not only different solvents (ethanol, methanol, 2-propanol, etc.), but also non-standard methods of activating the crystallization process. To do this, experiments on recrystallization from water under an ultrasonic field effect were carried out. It should be noted that under normal conditions, 4-[(benzyl- 
Table 1

Selected geometrical parameters $\left(\AA{ }^{\circ}{ }^{\circ}\right)$ for the cations in salts $\mathbf{I}$ and II.

\begin{tabular}{llll}
\hline Parameter & $\mathbf{I}$ & $\mathbf{I I} A$ & $\mathbf{I I} B$ \\
\hline $\mathrm{N} 1-\mathrm{C} 2$ & $1.338(10)$ & $1.327(19)$ & $1.32(2)$ \\
$\mathrm{N} 1-\mathrm{C} 6$ & $1.324(11)$ & $1.35(2)$ & $1.313(18)$ \\
$\mathrm{N} 2-\mathrm{C} 7-\mathrm{C} 4-\mathrm{C} 3$ & $18.1(13)$ & $-16(2)$ & $18(2)$ \\
$\mathrm{C} 7-\mathrm{N} 2-\mathrm{C} 8-\mathrm{C} 9$ & $-75.0(11)$ & $-81(2)$ & $178.3(14)$ \\
$\mathrm{N} 2-\mathrm{C} 8-\mathrm{C} 9-\mathrm{C} 10$ & $-77.6(11)$ & $-61.6(18)$ & $-53(2)$ \\
$\mathrm{H} 2 \cdots \mathrm{H} 3$ & 2.09 & 2.14 & 2.11 \\
$\mathrm{C} 3 \cdots \mathrm{H} 2$ & 2.55 & 2.61 & 2.57 \\
\hline
\end{tabular}

amino) carbonyl]-1-methylpyridinium iodide does not dissolve in water. As result, we did not obtain any new polymorphic modifications of this salt, but two compounds with cationiodine ratios different from the equimolar [1:2 (salt I) and 1:3 (salt II)] were obtained.

\section{Structural commentary}

The crystal structures of the salts under study consist of the same 4-[(benzylamino)carbonyl]-1-methylpyridinium cation $\left(\mathrm{C}_{14} \mathrm{H}_{15} \mathrm{~N}_{2} \mathrm{O}^{+}\right)$and different anions. There is one cation, one iodide anion and half of the neutral $\mathrm{I}_{2}$ molecule in the asymmetric unit of compound I (Fig. 1, left). The neutral $\mathrm{I}_{2}$ molecule is located in a special position in relation to the symmetry centre coinciding with the midpoint of the I-I bond. Thus, the cation:iodine atoms ratio is 1:2 in compound $\mathbf{I}$. The asymmetric unit of compound II contains two cations $(A$ and $B$ ), one triiodide anion $\left(\mathrm{I}_{\mathbf{3}}{ }^{-}\right)$and two halves of triiodide anions located on special positions in relation to the symmetry centre (Fig. 1, right). The cation:iodine atoms ration is 1:3 in compound II.

The positive charge of the cation is localized at the quaternized nitrogen atom of the pyridine ring. This results in the $\mathrm{N} 1-\mathrm{C} 6$ and $\mathrm{N} 1-\mathrm{C} 2$ bond elongation (Table 1). The carbamide group is non-coplanar to the plane of the aromatic ring (as evidenced by the $\mathrm{N} 2-\mathrm{C} 7-\mathrm{C} 4-\mathrm{C} 3$ torsion angles; Table 1) as a result of steric repulsion between them [with short $\mathrm{H} 2 \cdots \mathrm{H} 3$ and $\mathrm{H} 2 \cdots \mathrm{C} 3$ contacts (as compared to the van der Waals radii sums; Zefirov, 1997) of 2.34 and $2.87 \AA$,
Table 2

Hydrogen-bond geometry $\left(\AA,^{\circ}\right)$ for $\mathbf{I}$.

\begin{tabular}{lllll}
\hline$D-\mathrm{H} \cdots A$ & $D-\mathrm{H}$ & $\mathrm{H} \cdots A$ & $D \cdots A$ & $D-\mathrm{H} \cdots A$ \\
\hline $\mathrm{N} 2-\mathrm{H} 2 \cdots \mathrm{I} 2$ & 0.86 & 2.84 & $3.632(7)$ & 154 \\
$\mathrm{C} 2-\mathrm{H} 2 A \cdots \mathrm{I} 2^{\mathrm{i}}$ & 0.93 & 3.18 & $4.053(9)$ & 158 \\
$\mathrm{C} 1-\mathrm{H} 1 B \cdots \mathrm{I} 2^{\mathrm{i}}$ & 0.96 & 3.11 & $3.992(9)$ & 153 \\
$\mathrm{C} 1-\mathrm{H} 1 C \cdots \mathrm{I} 2^{\mathrm{ii}}$ & 0.96 & 2.96 & $3.908(9)$ & 171 \\
$\mathrm{C} 1-\mathrm{H} 1 A \cdots \mathrm{I} 1^{\mathrm{iii}}$ & 0.96 & 3.00 & $3.824(10)$ & 145 \\
$\mathrm{C} 5-\mathrm{H} 5 \cdots \mathrm{O} 1^{\text {iv }}$ & 0.93 & 2.59 & $3.328(11)$ & 136 \\
$\mathrm{C} 8-\mathrm{H} 8 B \cdots \mathrm{C} 11^{\mathrm{v}}$ & 0.97 & 2.80 & $3.590(15)$ & 140 \\
$\mathrm{C} 8-\mathrm{H} 8 B \cdots \mathrm{C} 10^{\mathrm{v}}$ & 0.97 & 2.76 & $3.694(14)$ & 162
\end{tabular}

Symmetry codes: (i) $\quad-x+1,-y+1,-z+1 ; \quad$ (ii) $\quad x-\frac{1}{2},-y+\frac{3}{2}, z-\frac{1}{2}$; $\quad$ (iii) $x-\frac{1}{2},-y+\frac{1}{2}, z-\frac{1}{2}$; (iv) $-x,-y+1,-z+1 ;$ (v) $-x+\frac{1}{2}, y-\frac{1}{2},-z+\frac{3}{2}$.

Table 3

Hydrogen-bond geometry $\left(\AA,^{\circ}\right)$ for II.

\begin{tabular}{lllll}
\hline$D-\mathrm{H} \cdots A$ & $D-\mathrm{H}$ & $\mathrm{H} \cdots A$ & $D \cdots A$ & $D-\mathrm{H} \cdots A$ \\
\hline $\mathrm{N} 2 A-\mathrm{H} 2 A \cdots \mathrm{O} 1 B$ & 0.86 & 2.02 & $2.846(14)$ & 160 \\
$\mathrm{C} 3 A-\mathrm{H} 3 A \cdots \mathrm{O} 1 B$ & 0.93 & 2.53 & $3.381(18)$ & 152 \\
$\mathrm{C} 2 A-\mathrm{H} 2 A A \cdots \mathrm{I} 3$ & 0.93 & 3.08 & $3.998(17)$ & 169 \\
$\mathrm{C} 1 A-\mathrm{H} 1 A C \cdots \mathrm{C} 12 A^{\mathrm{i}}$ & 0.96 & 2.72 & $3.62(2)$ & 158 \\
$\mathrm{C} 1 A-\mathrm{H} 1 A A \cdots \mathrm{I} 7^{\mathrm{i}}$ & 0.96 & 3.09 & $3.966(19)$ & 153 \\
$\mathrm{~N} 2 B-\mathrm{H} 2 B \cdots \mathrm{O} 1 A^{\mathrm{ii}}$ & 0.86 & 2.13 & $2.986(14)$ & 176 \\
$\mathrm{C} 3 B-\mathrm{H} 3 B \cdots \mathrm{O} 1 A^{\mathrm{ii}}$ & 0.93 & 2.21 & $3.060(17)$ & 151 \\
$\mathrm{C} 2 B-\mathrm{H} 2 B A \cdots \mathrm{C} 12 A^{\text {ii }}$ & 0.93 & 2.85 & $3.72(2)$ & 156 \\
$\mathrm{C} 1 B-\mathrm{H} 1 B B \cdots \mathrm{I} 7^{\text {iv }}$ & 0.96 & 3.07 & $3.819(18)$ & 136 \\
$\mathrm{C} 6 B-\mathrm{H} 6 B \cdots 4^{\mathrm{v}}$ & 0.93 & 3.12 & $4.019(17)$ & 164 \\
\hline
\end{tabular}

Symmetry codes: (i) $x,-y+\frac{3}{2}, z-\frac{1}{2}$; (ii) $x+1, y, z$; (iii) $-x+1,-y+1,-z+1$; (iv) $-x,-y+1,-z+1 ;(\mathrm{v})-x, y-\frac{1}{2},-z+\frac{1}{2}$.

respectively]. The cations in the two compounds under study differ in the conformation of the benzyl substituent. The phenyl fragment of the benzyl substituent is located in a $-s c$ position relatively to the $\mathrm{C} 7-\mathrm{N} 2$ bond in $\mathbf{I}$ or in a $+s c$ position in molecule $A$ and an ap position in molecule $B$ of II ( $c f$ the $\mathrm{C} 7-\mathrm{N} 2-\mathrm{C} 8-\mathrm{C} 9$ torsion angles in Table 1$)$. The aromatic ring is turned relative to the carbamide fragment (see the $\mathrm{N} 2-\mathrm{C} 8-\mathrm{C} 9-\mathrm{C} 10$ torsion angles).

\section{Supramolecular features}

The main difference in the crystal structures of the studied salts is the participation of the carbamide group in inter-
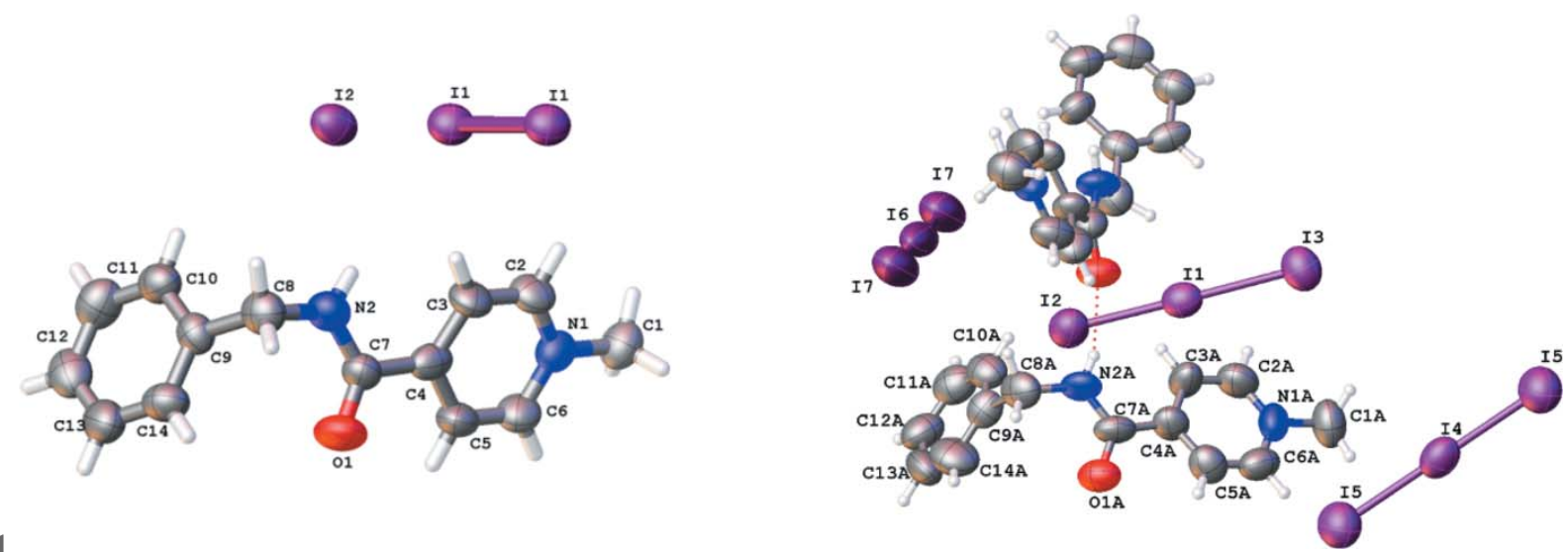

Figure 1

Molecular structures of I (on the left) and II (on the right), showing the atom labeling scheme. Displacement ellipsoids are drawn at the $50 \%$ probability level. 


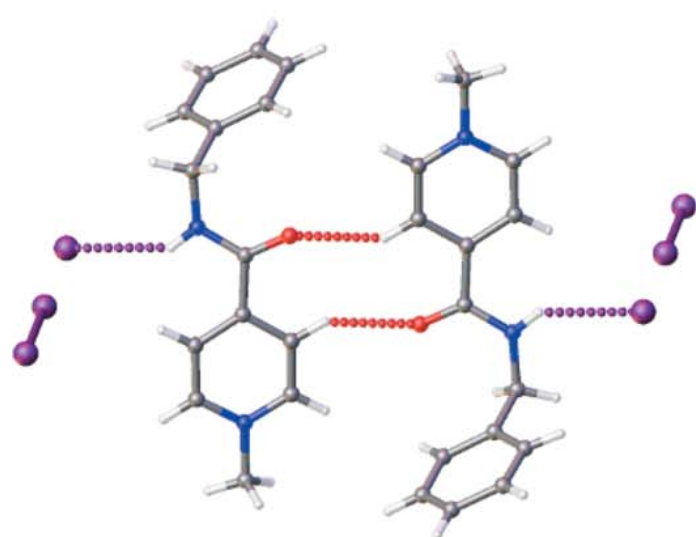

Figure 2

Hydrogen bond formation in structure I (on the left) and II (on the right).

molecular interactions. In the structure of $\mathbf{I}$, the carbamide group participates in the $\mathrm{N}-\mathrm{H} \cdots \mathrm{I}^{\prime}$ hydrogen bond between the cation and the anion, while the carbonyl oxygen atom acts as an acceptor in the very weak $\mathrm{C} 5-\mathrm{H}$. . O $1^{\prime}$ intermolecular interaction (Fig. 2, left; Table 2). In the structure of II, the carbamide group participates in the $\mathrm{N}-\mathrm{H} \cdots \mathrm{O}^{\prime}$ hydrogen bonds between the cations (Fig. 2, right; Table 3). As a result, chains in the [100] crystallographic direction are formed. The triiodide anions occupy voids between neighbouring chains in the crystal. In addition, a set of weak $\mathrm{C}-\mathrm{H} \cdots \mathrm{I}$ and $\mathrm{C}-\mathrm{H} \cdots \pi$ hydrogen bonds are found in both structures (Tables 2 and 3 ).

In the structure of II, the $A$ and $B$ cations form stacking dimers as a result of the interaction of the aromatic systems of the pyridine and benzene rings [the distance between the planes of aromatic cycles is 3.45 (1) $\AA$, slippage $1.119 \AA$ ).

\section{Hirshfeld surface analysis}

Intermolecular interactions can be analyzed using Hirshfeld surface analysis and 2D fingerprint plots (Turner et al., 2017). The Hirshfeld surfaces were calculated for the cations found in two structures under study using a standard high surface resolution, mapped over $d_{\text {norm }}$ (Fig. 3). The red spots, corresponding to contacts that are shorter than the van der Waals radii sum of the closest atoms, are observed at the hydrogen atom of the amino group. At the carbonyl group, red spots are found only in the cations of II. The two-dimensional finger-
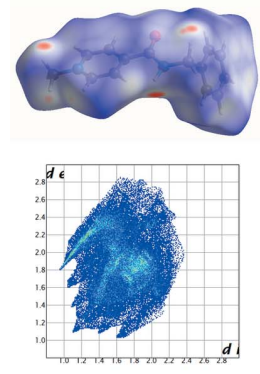

Structure I
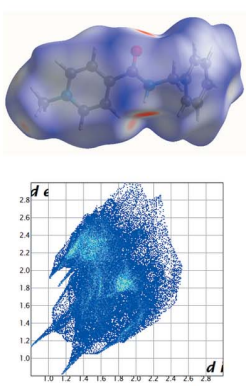

Structure II, molecule A
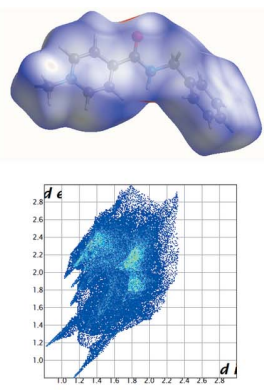

Structure II, molecule B
Figure 3

Hirshfeld surfaces mapped over $d_{\text {norm. }}$ (at the top) and two-dimensional fingerprint plots (at the bottom) of cation in structure I and II.

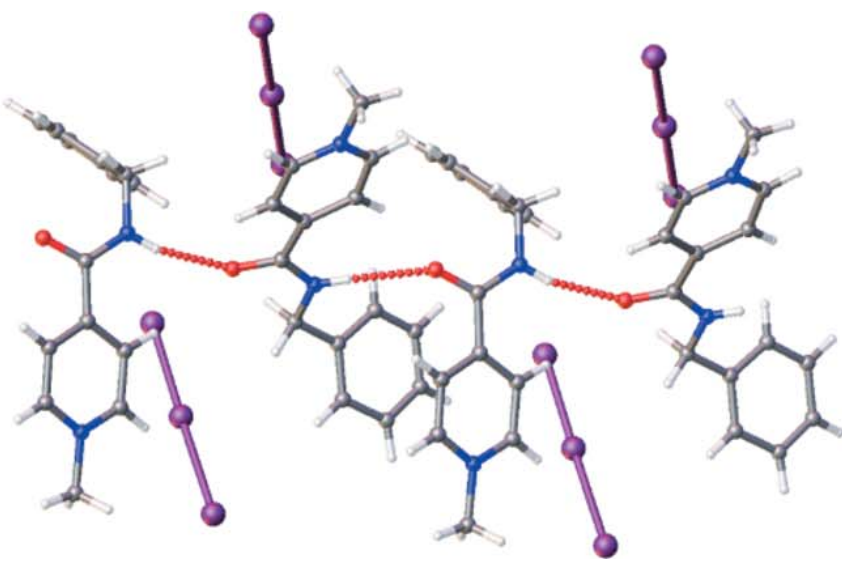

print plots show that the hydrogen bonds in II are stronger (note the sharp spikes in Fig. 3).

To compare intermolecular interactions of different types in more quantitative way, their contributions to the total Hirshfeld surfaces were analysed (Fig. 4). The main contribution is provided by $\mathrm{H} \cdots \mathrm{H}$ short contacts $(44.9 \%$ for $\mathbf{I}, 45 \%$ for cation $A$ and $36.8 \%$ for cation $B$ in II). The contribution of the $\mathrm{I} \cdots \mathrm{H} /$ $\mathrm{H}$...I short contacts is also significant $[17.3 \%$ in $\mathbf{I}, 21.7 \%$ (molecule $A$ ) and $25.5 \%$ (molecule $B$ ) in $\mathbf{I I}]$, as is that of the C. $\cdot \mathrm{H} / \mathrm{H} \cdots \mathrm{C}$ interactions $[17.2 \%$ in $\mathbf{I}, 15.5 \%$ (molecule $A$ ) and $10.7 \%$ (molecule $B$ ) in II]. Surprisingly, the contributions of the $\mathrm{O} \cdots \mathrm{H} / \mathrm{H} \cdots \mathrm{O}$ interactions are very similar in the two structures [9.7\% in I, 9.5\% (molecule $A$ ) and $9.6 \%$ (molecule $B)$ in II] despite the stronger $\mathrm{N}-\mathrm{H} \ldots \mathrm{O}$ hydrogen bonds in the structure of II.

\section{Database survey}

A search of the Cambridge Structural Database (Version 5.42, update of November 2020; Groom et al., 2016) revealed the structure of the AmI salt with an equimolar cation:iodine atoms ratio (refcode BEBFIA; Drebushchak et al., 2017). A comparison of the cation conformations showed its flexibility resulting from rotation about the $\mathrm{N}-\mathrm{Csp}^{3}$ and $\mathrm{Csp}{ }^{3}-\mathrm{Car}$ bonds.

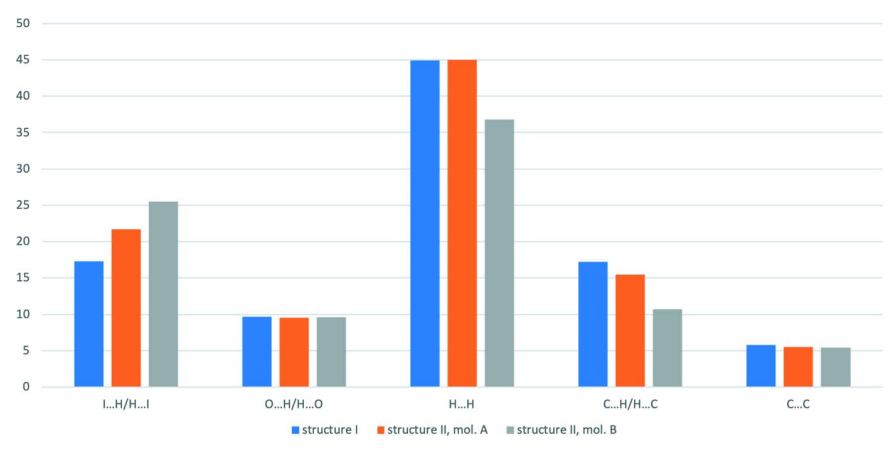

Figure 4

Relative contributions of the strongest intermolecular interactions (in \%) to the total Hirshfeld surface of cation in two iodide salts. 
Table 4

Experimental details.

I

Crystal data

Chemical formula

$M_{\text {r }}$

Crystal system, space group

Temperature (K)

$a, b, c(\AA)$

$\beta\left({ }^{\circ}\right)$

$V\left(\AA^{3}\right)$

Z

Radiation type

$\mu\left(\mathrm{mm}^{-1}\right)$

Crystal size (mm)

Data collection

Diffractometer

Absorption correction

$T_{\min }, T_{\max }$

No. of measured, independent and

observed $[I>2 \sigma(I)]$ reflections

$R_{\text {int }}$

$(\sin \theta / \lambda)_{\max }\left(\AA^{-1}\right)$

Refinemen

$R\left[F^{2}>2 \sigma\left(F^{2}\right)\right], w R\left(F^{2}\right), S$

No. of reflections

No. of parameters

$\mathrm{H}$-atom treatment

$\Delta \rho_{\max }, \Delta \rho_{\min }\left(\mathrm{e} \AA^{-3}\right)$

\author{
$\mathrm{C}_{14} \mathrm{H}_{15} \mathrm{~N}_{2} \mathrm{O}^{+} \cdot \mathrm{I}^{-} \cdot 0.5 \mathrm{I}_{2}$ \\ 481.08 \\ Monoclinic, $P 2_{1} / n$ \\ 293 \\ 14.407 (3), 8.8491 (10), 14.555 (4) \\ $119.63(3)$ \\ $1613.0(7)$ \\ 4 \\ Mo $K \alpha$ \\ 3.89 \\ $0.60 \times 0.10 \times 0.05$
}

Xcalibur, Sapphire3

Multi-scan (CrysAlis PRO; Rigaku OD, 2018)

$0.159,1.000$

11491, 3698, 1941

0.083

0.650

$0.053,0.157,1.03$

3698

173

H-atom parameters constrained

$0.90,-0.90$
II

\author{
$\mathrm{C}_{14} \mathrm{H}_{15} \mathrm{~N}_{2} \mathrm{O}^{+} \cdot \mathrm{I}_{3}{ }^{-}$ \\ 608.61 \\ Monoclinic, $P 2_{1} / c$ \\ 293 \\ 9.914 (2), 27.805 (4), 14.113 (3) \\ $107.83(2)$ \\ 3703.4 (12) \\ 8 \\ Mo $K \alpha$ \\ 5.07 \\ $0.03 \times 0.03 \times 0.02$
}

Xcalibur, Sapphire3

Multi-scan (CrysAlis PRO; Rigaku OD, 2018)

$0.347,1.000$

21040, 6496, 2548

0.124

0.595

$0.065,0.187,0.97$

6496

371

$\mathrm{H}$-atom parameters constrained

$0.70,-0.77$

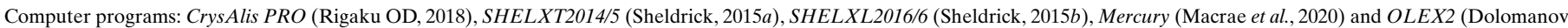
et al., 2009).

\section{Synthesis and crystallization}

Benzylamide isonicotinic acid (124 g, $0.585 \mathrm{~mol})$ and $270 \mathrm{~mL}$ of $90 \%$ ethanol were loaded into a glass flask. The obtained solution was heated to a temperature of 313-314 K, and then methyl iodide $(91 \mathrm{~g}, 0.641 \mathrm{~mol})$ was added dropwise. The reaction was stirred at a temperature of $313-314 \mathrm{~K}$ for $1 \mathrm{~h}$, heated to boiling and boiled for $1 \mathrm{~h}$. The reaction spontaneously cooled to a temperature of $313 \mathrm{~K}$, then to a temperature of $283-288 \mathrm{~K}$ in a cooling water bath, and was stirred for $1.5 \mathrm{~h}$ at this temperature. The reaction mixture was filtered and the precipitate rinsed on the filter twice with $60 \mathrm{~mL}$ of cooled $96 \%$ ethanol. The product was dried at $313 \mathrm{~K}$ for $12 \mathrm{~h}$. Yield: $145.5 \mathrm{~g}$ of crude 4-[(benzylamino)carbonyl]-1methylpyridinium iodide ( $88 \%)$; yellow crystals.

$145.5 \mathrm{~g}$ of crude 4-[(benzylamino)carbonyl]-1-methylpyridinium iodide were dissolved in $450 \mathrm{~mL}$ of water under ultrasonic activation. The reaction was heated to boiling temperature, stirred at boiling for $30 \mathrm{~min}$ and filtered. The obtained solution was cooled slowly and evaporated for three weeks. The rod-shaped crystals of $\mathbf{I}$ and block-shaped crystals of II crystallized almost simultaneously.

\section{Refinement}

Crystal data, data collection and structure refinement details are summarized in Table 4. Despite the presence of iodine atoms, crystals of salt II diffracted poorly due to their small size. All of the hydrogen atoms were located in difference-
Fourier maps. Then, hydrogen atoms were refined as riding (AFIX 33 and 137 commands) with $\mathrm{C}-\mathrm{H}=0.96 \AA, U_{\text {iso }}(\mathrm{H})=$ $1.5 U_{\text {eq }}(\mathrm{C})$ for methyl groups (AFIX 43) and $\mathrm{C}_{\mathrm{ar}}-\mathrm{H}=0.93 \AA$, $U_{\text {iso }}(\mathrm{H})=1.2 U_{\text {eq }}(\mathrm{C})$ for aromatic rings (AFIX 23) and $\mathrm{Csp}^{2}-$ $\mathrm{H}=0.97 \AA, U_{\text {iso }}(\mathrm{H})=1.2 U_{\text {eq }}(\mathrm{C})$ for the methylene fragment.

\section{Powder diffraction characterization}

A powder diffraction pattern of salt II was registered using a Siemens D500 powder diffractometer $(\mathrm{Cu} \mathrm{K \alpha}$ radiation, Bragg-Brentano geometry, curved graphite monochromator on the counter arm, $4<2 \theta<60^{\circ}, D 2 \theta=0.02^{\circ}$, time per step of $2 \mathrm{~s}$ ). The Rietveld refinement of the obtained pattern (Fig. 5, left) was carried out with FULLPROF (Rodriguez-Carvajal,
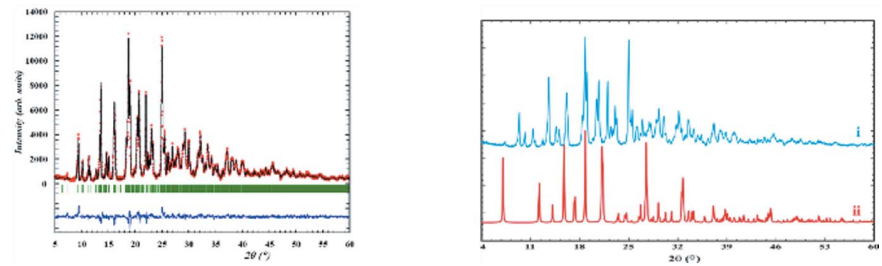

Figure 5

Final Rietveld plots for II (on the left). Observed data points are indicated by red circles, the best-fit profile (black upper trace) and the difference pattern (blue lower trace) are shown as solid lines. The vertical green bars correspond to the Bragg positions of peaks. The calculated powder pattern for $\mathbf{I}$ is shown on the right. 
2001) and WINPLOTR (Roisnel \& Rodriguez-Carvajal, 2000) using an external standard (NIST SRM1976) for the calculation of the instrumental profile function and the single-crystal results as the structure model for the refinement. A powder pattern for salt I was not obtained because of the small amount of the crystal sample. For comparison, Fig. 5 (right) shows the pattern calculated for salt $\mathbf{I}$.

\section{Acknowledgements}

The authors are grateful to Farmak JSC for support.

\section{Funding information}

Funding for this research was provided by: National Academy of Sciences of Ukraine (grant No. 0120U102660).

\section{References}

Bernstein, J. (2002). Polymorphism in Molecular Crystals. Oxford: Clarendon Press.

Boltz, D., Peng, X., Muzzio, M., Dash, P., Thomas, P. G. \& Margitich, V. (2018). Antivir. Chem. Chemother. 26 https://doi.org/10.1177/ 2040206618811416.

Brittain, H. G. (2009). Polymorphism in pharmaceutical solids, 2nd ed. New York: Informa.

Buhtiarova, T. A., Danilenko, V. P., Homenko, V. S., Shatyrkina, T. V. \& Yadlovsky, O. E. (2003). Ukrainian Med. J. 33, 72-74.

Dolomanov, O. V., Bourhis, L. J., Gildea, R. J., Howard, J. A. K. \& Puschmann, H. (2009). J. Appl. Cryst. 42, 339-341.
Drebushchak, T. N., Kryukov, Y. A., Rogova, A. I. \& Boldyreva, E. V. (2017). Acta Cryst. E73, 967-970.

Frolov, A. F., Frolov, V. M., Buhtiarova, T. A. \& Danilenko, V. P. (2004). Ukrainian Med. J. 39, 69-74.

Groom, C. R., Bruno, I. J., Lightfoot, M. P. \& Ward, S. C. (2016). Acta Cryst. B72, 171-179.

Hilfiker, R. (2006). Polymorphism in the Pharmaceutical Industry. Weinheim: John Wiley \& Sons.

Macrae, C. F., Sovago, I., Cottrell, S. J., Galek, P. T. A., McCabe, P., Pidcock, E., Platings, M., Shields, G. P., Stevens, J. S., Towler, M. \& Wood, P. A. (2020). J. Appl. Cryst. 53, 226-235.

Rigaku OD (2018). CrysAlis PRO. Rigaku Oxford Diffraction, Yarnton, England.

Rodríguez-Carvajal, J. (2001). Commission on Powder Diffraction (IUCr) Newsletter, 26, 12-19.

Roisnel, T. \& Rodríguez-Carvajal, J. (2000). WinPLOTR, a Windows tool for powder diffraction patterns analysis. Mater. Sci. Forum, Proc. 7th Europ. Powder Diff. Conf. (EPDIC 7), edited by R. Delhez \& E. J. Mittenmeijer, pp. 118-123.

Sheldrick, G. M. (2015a). Acta Cryst. A71, 3-8.

Sheldrick, G. M. (2015b). Acta Cryst. A71, 3-8.

Turner, M. J., McKinnon, J. J., Wolff, S. K., Grimwood, D. J., Spackman, P. R., Jayatilaka, D. \& Spackman, M. A. (2017). CrystalExplorer17. University of Western Australia. http://Hirshfeldsurface.net

Velthuis, A. J. W. te, Zubkova, T. G., Shaw, M., Mehle, A., Boltz, D., Gmeinwieser, N., Stammer, H., Milde, J., Müller, L. \& Margitich, V. (2020). Antimicrobial Agents and Chemotherapy, 64, https:// doi.org/10.1128/AAC.02605-20.

Zefirov, Yu. V. (1997). Kristallografiya, 42, 936-958. 


\section{supporting information}

Acta Cryst. (2021). E77, 1219-1223 [https://doi.org/10.1107/S2056989021011300]

\section{Salts of 4-[(benzylamino)carbonyl]-1-methylpyridinium and iodide anions with different cation:iodine stoichiometric ratios}

Vitalii V. Rudiuk, Anna M. Shaposhnyk, Vyacheslav M. Baumer, Igor A. Levandovskiy and

\section{Svitlana V. Shishkina}

\section{Computing details}

For both structures, data collection: CrysAlis PRO (Rigaku OD, 2018); cell refinement: CrysAlis PRO (Rigaku OD, 2018); data reduction: CrysAlis PRO (Rigaku OD, 2018); program(s) used to solve structure: SHELXT2014/5 (Sheldrick, 2015a); program(s) used to refine structure: SHELXL2016/6 (Sheldrick, 2015b); molecular graphics: Mercury (Macrae et al., 2020); software used to prepare material for publication: OLEX2 (Dolomanov et al., 2009).

4-[(Benzylamino)carbonyl]-1-methylpyridinium iodide-iodine (2/1) (I)

\section{Crystal data}

$\mathrm{C}_{14} \mathrm{H}_{15} \mathrm{~N}_{2} \mathrm{O}^{+} \cdot \mathrm{I}^{-} \cdot 0.5 \mathrm{I}_{2}$

$M_{r}=481.08$

Monoclinic, $P 2_{1} / n$

$a=14.407(3) \AA$

$b=8.8491(10) \AA$

$c=14.555(4) \AA$

$\beta=119.63(3)^{\circ}$

$V=1613.0(7) \AA^{3}$

$Z=4$

\section{Data collection}

Xcalibur, Sapphire3 diffractometer

Radiation source: Enhance (Mo) X-ray Source Detector resolution: 16.1827 pixels $\mathrm{mm}^{-1}$ $\omega$ scans

Absorption correction: multi-scan

(CrysAlisPro; Rigaku OD, 2018)

$T_{\min }=0.159, T_{\max }=1.000$

\section{Refinement}

Refinement on $F^{2}$

Least-squares matrix: full

$R\left[F^{2}>2 \sigma\left(F^{2}\right)\right]=0.053$

$w R\left(F^{2}\right)=0.157$

$S=1.03$

3698 reflections

173 parameters

0 restraints
$F(000)=908$

$D_{\mathrm{x}}=1.981 \mathrm{Mg} \mathrm{m}^{-3}$

Mo $K \alpha$ radiation, $\lambda=0.71073 \AA$

Cell parameters from 928 reflections

$\theta=3.6-21.8^{\circ}$

$\mu=3.89 \mathrm{~mm}^{-1}$

$T=293 \mathrm{~K}$

Stick, red

$0.60 \times 0.10 \times 0.05 \mathrm{~mm}$

11491 measured reflections

3698 independent reflections

1941 reflections with $I>2 \sigma(I)$

$R_{\text {int }}=0.083$

$\theta_{\text {max }}=27.5^{\circ}, \theta_{\min }=3.2^{\circ}$

$h=-18 \rightarrow 18$

$k=-11 \rightarrow 11$

$l=-18 \rightarrow 18$

Hydrogen site location: inferred from neighbouring sites

$\mathrm{H}$-atom parameters constrained

$w=1 /\left[\sigma^{2}\left(F_{\mathrm{o}}{ }^{2}\right)+(0.0416 P)^{2}\right]$

where $P=\left(F_{\mathrm{o}}{ }^{2}+2 F_{\mathrm{c}}{ }^{2}\right) / 3$

$(\Delta / \sigma)_{\max }=0.001$

$\Delta \rho_{\max }=0.90 \mathrm{e} \AA^{-3}$

$\Delta \rho_{\min }=-0.89$ e $\AA^{-3}$ 


\section{Special details}

Geometry. All esds (except the esd in the dihedral angle between two 1.s. planes) are estimated using the full covariance matrix. The cell esds are taken into account individually in the estimation of esds in distances, angles and torsion angles; correlations between esds in cell parameters are only used when they are defined by crystal symmetry. An approximate (isotropic) treatment of cell esds is used for estimating esds involving l.s. planes.

Fractional atomic coordinates and isotropic or equivalent isotropic displacement parameters $\left(\AA^{2}\right)$

\begin{tabular}{|c|c|c|c|c|}
\hline & $x$ & $y$ & $z$ & $U_{\text {iso }} * / U_{\text {eq }}$ \\
\hline I1 & $0.51630(5)$ & $0.11900(6)$ & $0.57075(6)$ & $0.0696(2)$ \\
\hline $\mathrm{I} 2$ & $0.55433(5)$ & $0.39112(7)$ & $0.74152(6)$ & 0.0738 (3) \\
\hline $\mathrm{O} 1$ & $0.1044(5)$ & $0.3003(8)$ & $0.5772(6)$ & $0.090(2)$ \\
\hline N1 & $0.1782(6)$ & $0.6060(7)$ & $0.3371(6)$ & $0.0614(18)$ \\
\hline N2 & $0.2820(6)$ & $0.2698(8)$ & $0.6521(6)$ & 0.0652 (19) \\
\hline $\mathrm{H} 2$ & 0.338519 & 0.295372 & 0.650010 & $0.078^{*}$ \\
\hline $\mathrm{C} 1$ & $0.1732(8)$ & $0.7074(10)$ & $0.2544(8)$ & $0.075(3)$ \\
\hline H1A & 0.127757 & 0.663816 & 0.186085 & $0.113 *$ \\
\hline H1B & 0.243679 & 0.720760 & 0.264125 & $0.113^{*}$ \\
\hline $\mathrm{H} 1 \mathrm{C}$ & 0.145051 & 0.803613 & 0.259012 & $0.113^{*}$ \\
\hline $\mathrm{C} 2$ & $0.2636(7)$ & $0.5182(10)$ & $0.3929(8)$ & 0.069 (3) \\
\hline $\mathrm{H} 2 \mathrm{~A}$ & 0.320082 & 0.521097 & 0.379074 & $0.083^{*}$ \\
\hline C3 & $0.2692(7)$ & $0.4235(10)$ & $0.4707(8)$ & $0.067(2)$ \\
\hline H3 & 0.327838 & 0.360259 & 0.506976 & $0.081 *$ \\
\hline $\mathrm{C} 4$ & $0.1885(6)$ & $0.4224(9)$ & $0.4944(8)$ & $0.062(2)$ \\
\hline $\mathrm{C} 5$ & $0.1012(7)$ & $0.5127(12)$ & $0.4336(8)$ & $0.078(3)$ \\
\hline H5 & 0.043510 & 0.511632 & 0.445534 & $0.093^{*}$ \\
\hline $\mathrm{C} 6$ & 0.0979 (7) & $0.6028(10)$ & $0.3569(8)$ & $0.070(3)$ \\
\hline H6 & 0.038316 & 0.663303 & 0.317556 & $0.084^{*}$ \\
\hline $\mathrm{C} 7$ & $0.1885(6)$ & $0.3260(10)$ & $0.5791(7)$ & $0.057(2)$ \\
\hline $\mathrm{C} 8$ & $0.2932(8)$ & $0.1666(10)$ & $0.7357(8)$ & $0.073(3)$ \\
\hline H8A & 0.362531 & 0.117925 & 0.766315 & $0.087 *$ \\
\hline H8B & 0.239142 & 0.088488 & 0.704706 & $0.087^{*}$ \\
\hline C9 & $0.2828(7)$ & $0.2439(8)$ & $0.8213(7)$ & $0.057(2)$ \\
\hline $\mathrm{C} 10$ & $0.3668(7)$ & $0.3281(10)$ & $0.8984(8)$ & $0.068(2)$ \\
\hline H10 & 0.431231 & 0.333813 & 0.898376 & $0.082 *$ \\
\hline $\mathrm{C} 11$ & $0.3548(9)$ & $0.4014(10)$ & $0.9732(9)$ & $0.081(3)$ \\
\hline H11 & 0.410137 & 0.461576 & 1.022065 & $0.097^{*}$ \\
\hline $\mathrm{C} 12$ & $0.2622(9)$ & $0.3890(10)$ & $0.9788(9)$ & $0.078(3)$ \\
\hline H12 & 0.255082 & 0.439378 & 1.031040 & $0.094^{*}$ \\
\hline $\mathrm{C} 13$ & $0.1810(9)$ & $0.3010(13)$ & 0.9058 (9) & $0.083(3)$ \\
\hline H13 & 0.118664 & 0.289920 & 0.909431 & $0.099 *$ \\
\hline $\mathrm{C} 14$ & $0.1904(7)$ & $0.2294(10)$ & $0.8280(9)$ & $0.071(3)$ \\
\hline H14 & 0.134482 & 0.170291 & 0.778882 & $0.085^{*}$ \\
\hline
\end{tabular}

Atomic displacement parameters $\left(\AA^{2}\right)$

\begin{tabular}{lllllll}
\hline & $U^{11}$ & $U^{22}$ & $U^{33}$ & $U^{12}$ & $U^{13}$ & $U^{23}$ \\
\hline I1 & $0.0651(4)$ & $0.0655(4)$ & $0.0790(5)$ & $0.0033(3)$ & $0.0364(4)$ & $-0.0008(3)$
\end{tabular}




$\begin{array}{lllllll}\mathrm{I} 2 & 0.0669(4) & 0.0799(4) & 0.0809(5) & -0.0121(3) & 0.0413(4) & -0.0181(3) \\ \mathrm{O} 1 & 0.050(3) & 0.118(5) & 0.100(6) & -0.008(3) & 0.036(4) & 0.012(5) \\ \mathrm{N} 1 & 0.060(4) & 0.060(4) & 0.056(5) & 0.006(3) & 0.023(4) & -0.002(3) \\ \mathrm{N} 2 & 0.058(4) & 0.068(4) & 0.074(6) & -0.007(3) & 0.036(4) & 0.003(4) \\ \text { C1 } & 0.081(6) & 0.070(6) & 0.062(7) & 0.008(5) & 0.025(5) & 0.008(5) \\ \text { C2 } & 0.066(6) & 0.070(6) & 0.080(8) & 0.013(4) & 0.042(6) & -0.002(5) \\ \text { C3 } & 0.057(5) & 0.083(6) & 0.073(7) & 0.018(4) & 0.040(5) & 0.006(5) \\ \text { C4 } & 0.051(5) & 0.066(5) & 0.060(6) & 0.003(4) & 0.021(4) & -0.019(4) \\ \text { C5 } & 0.048(5) & 0.121(8) & 0.067(7) & 0.008(5) & 0.030(5) & 0.004(6) \\ \text { C6 } & 0.059(5) & 0.075(6) & 0.073(7) & 0.017(4) & 0.031(5) & 0.005(5) \\ \text { C7 } & 0.050(4) & 0.063(5) & 0.060(6) & -0.006(4) & 0.029(4) & -0.002(4) \\ \text { C8 } & 0.075(6) & 0.066(5) & 0.077(7) & 0.001(5) & 0.037(6) & 0.013(5) \\ \text { C9 } & 0.067(5) & 0.048(4) & 0.060(6) & 0.005(4) & 0.035(5) & 0.006(4) \\ \text { C10 } & 0.059(5) & 0.072(6) & 0.072(7) & -0.004(4) & 0.033(5) & 0.010(5) \\ \text { C11 } & 0.086(7) & 0.068(6) & 0.074(8) & -0.012(5) & 0.028(6) & -0.002(5) \\ \text { C12 } & 0.092(8) & 0.072(6) & 0.073(8) & 0.027(5) & 0.043(7) & 0.017(5) \\ \text { C13 } & 0.075(6) & 0.106(8) & 0.070(7) & 0.014(6) & 0.039(6) & 0.019(6) \\ \text { C14 } & 0.053(5) & 0.075(6) & 0.083(8) & -0.003(4) & 0.032(5) & 0.016(5) \\ & & & & & & \end{array}$

Geometric parameters $\left(\AA,{ }^{o}\right)$

\begin{tabular}{llll}
\hline $\mathrm{I} 1-\mathrm{I} 1 \mathrm{i}$ & $2.8182(13)$ & $\mathrm{C} 5-\mathrm{C} 6$ & $1.353(13)$ \\
$\mathrm{O} 1-\mathrm{C} 7$ & $1.221(9)$ & $\mathrm{C} 5-\mathrm{H} 5$ & 0.9300 \\
$\mathrm{~N} 1-\mathrm{C} 6$ & $1.324(11)$ & $\mathrm{C} 6-\mathrm{H} 6$ & 0.9300 \\
$\mathrm{~N} 1-\mathrm{C} 2$ & $1.338(10)$ & $\mathrm{C} 8-\mathrm{C} 9$ & $1.494(12)$ \\
$\mathrm{N} 1-\mathrm{C} 1$ & $1.475(11)$ & $\mathrm{C} 8-\mathrm{H} 8 \mathrm{~A}$ & 0.9700 \\
$\mathrm{~N} 2-\mathrm{C} 7$ & $1.332(11)$ & $\mathrm{C} 8-\mathrm{H} 8 \mathrm{~B}$ & 0.9700 \\
$\mathrm{~N} 2-\mathrm{C} 8$ & $1.465(11)$ & $\mathrm{C} 9-\mathrm{C} 14$ & $1.387(11)$ \\
$\mathrm{N} 2-\mathrm{H} 2$ & 0.8600 & $\mathrm{C} 9-\mathrm{C} 10$ & $1.391(12)$ \\
$\mathrm{C} 1-\mathrm{H} 1 \mathrm{~A}$ & 0.9600 & $\mathrm{C} 10-\mathrm{C} 11$ & $1.350(14)$ \\
$\mathrm{C} 1-\mathrm{H} 1 \mathrm{~B}$ & 0.9600 & $\mathrm{C} 10-\mathrm{H} 10$ & 0.9300 \\
$\mathrm{C} 1-\mathrm{H} 1 \mathrm{C}$ & 0.9600 & $\mathrm{C} 11-\mathrm{C} 12$ & $1.381(14)$ \\
$\mathrm{C} 2-\mathrm{C} 3$ & $1.378(12)$ & $\mathrm{C} 11-\mathrm{H} 11$ & 0.9300 \\
$\mathrm{C} 2-\mathrm{H} 2 \mathrm{~A}$ & 0.9300 & $\mathrm{C} 12-\mathrm{C} 13$ & $1.369(15)$ \\
$\mathrm{C} 3-\mathrm{C} 4$ & $1.366(11)$ & $\mathrm{C} 12-\mathrm{H} 12$ & 1.9300 \\
$\mathrm{C} 3-\mathrm{H} 3$ & 0.9300 & $\mathrm{C} 13-\mathrm{C} 14$ & 0.9300 \\
$\mathrm{C} 4-\mathrm{C} 5$ & $1.380(12)$ & $\mathrm{C} 13-\mathrm{H} 13$ & 0.9300 \\
$\mathrm{C} 4-\mathrm{C} 7$ & $1.499(13)$ & $\mathrm{C} 14-\mathrm{H} 14$ & $123.3(8)$ \\
& & & $119.4(8)$ \\
$\mathrm{C} 6-\mathrm{N} 1-\mathrm{C} 2$ & $119.8(8)$ & $\mathrm{O} 1-\mathrm{C} 7-\mathrm{N} 2$ & $117.2(7)$ \\
$\mathrm{C} 6-\mathrm{N} 1-\mathrm{C} 1$ & $119.7(7)$ & $\mathrm{O} 1-\mathrm{C} 7-\mathrm{C} 4$ & $113.1(7)$ \\
$\mathrm{C} 2-\mathrm{N} 1-\mathrm{C} 1$ & $120.5(8)$ & $\mathrm{N} 2-\mathrm{C} 7-\mathrm{C} 4$ & 109.0 \\
$\mathrm{C} 7-\mathrm{N} 2-\mathrm{C} 8$ & $123.3(7)$ & $\mathrm{N} 2-\mathrm{C} 8-\mathrm{C} 9$ & 109.0 \\
$\mathrm{C} 7-\mathrm{N} 2-\mathrm{H} 2$ & 118.4 & $\mathrm{~N} 2-\mathrm{C} 8-\mathrm{H} 8 \mathrm{~A}$ & 109.0 \\
$\mathrm{C} 8-\mathrm{N} 2-\mathrm{H} 2$ & 118.4 & $\mathrm{C} 9-\mathrm{C} 8-\mathrm{H} 8 \mathrm{~A}$ & 109.0 \\
$\mathrm{~N} 1-\mathrm{C} 1-\mathrm{H} 1 \mathrm{~A}$ & 109.5 & $\mathrm{~N} 2-\mathrm{C} 8-\mathrm{H} 8 \mathrm{~B}$ & 107.8 \\
$\mathrm{~N} 1-\mathrm{C} 1-\mathrm{H} 1 \mathrm{~B}$ & 109.5 & $\mathrm{H} 8-\mathrm{C}-\mathrm{C} 8-\mathrm{H} 8 \mathrm{~B}$ & \\
$\mathrm{H} 1 \mathrm{~A}-\mathrm{C} 1-\mathrm{H} 1 \mathrm{~B}$ & 109.5 & & \\
& & &
\end{tabular}




\begin{tabular}{|c|c|c|c|}
\hline $\mathrm{N} 1-\mathrm{C} 1-\mathrm{H} 1 \mathrm{C}$ & 109.5 & $\mathrm{C} 14-\mathrm{C} 9-\mathrm{C} 10$ & $118.4(9)$ \\
\hline $\mathrm{H} 1 \mathrm{~A}-\mathrm{C} 1-\mathrm{H} 1 \mathrm{C}$ & 109.5 & $\mathrm{C} 14-\mathrm{C} 9-\mathrm{C} 8$ & $120.8(9)$ \\
\hline $\mathrm{H} 1 \mathrm{~B}-\mathrm{C} 1-\mathrm{H} 1 \mathrm{C}$ & 109.5 & $\mathrm{C} 10-\mathrm{C} 9-\mathrm{C} 8$ & $120.8(8)$ \\
\hline $\mathrm{N} 1-\mathrm{C} 2-\mathrm{C} 3$ & $120.9(8)$ & $\mathrm{C} 11-\mathrm{C} 10-\mathrm{C} 9$ & $120.0(9)$ \\
\hline $\mathrm{N} 1-\mathrm{C} 2-\mathrm{H} 2 \mathrm{~A}$ & 119.5 & $\mathrm{C} 11-\mathrm{C} 10-\mathrm{H} 10$ & 120.0 \\
\hline $\mathrm{C} 3-\mathrm{C} 2-\mathrm{H} 2 \mathrm{~A}$ & 119.5 & $\mathrm{C} 9-\mathrm{C} 10-\mathrm{H} 10$ & 120.0 \\
\hline $\mathrm{C} 4-\mathrm{C} 3-\mathrm{C} 2$ & $120.0(8)$ & $\mathrm{C} 10-\mathrm{C} 11-\mathrm{C} 12$ & $121.5(10)$ \\
\hline $\mathrm{C} 4-\mathrm{C} 3-\mathrm{H} 3$ & 120.0 & $\mathrm{C} 10-\mathrm{C} 11-\mathrm{H} 11$ & 119.3 \\
\hline $\mathrm{C} 2-\mathrm{C} 3-\mathrm{H} 3$ & 120.0 & $\mathrm{C} 12-\mathrm{C} 11-\mathrm{H} 11$ & 119.3 \\
\hline $\mathrm{C} 3-\mathrm{C} 4-\mathrm{C} 5$ & $116.9(9)$ & $\mathrm{C} 13-\mathrm{C} 12-\mathrm{C} 11$ & $118.7(10)$ \\
\hline $\mathrm{C} 3-\mathrm{C} 4-\mathrm{C} 7$ & $123.9(8)$ & $\mathrm{C} 13-\mathrm{C} 12-\mathrm{H} 12$ & 120.7 \\
\hline $\mathrm{C} 5-\mathrm{C} 4-\mathrm{C} 7$ & $119.1(8)$ & $\mathrm{C} 11-\mathrm{C} 12-\mathrm{H} 12$ & 120.7 \\
\hline $\mathrm{C} 6-\mathrm{C} 5-\mathrm{C} 4$ & $121.4(8)$ & $\mathrm{C} 14-\mathrm{C} 13-\mathrm{C} 12$ & $120.7(10)$ \\
\hline $\mathrm{C} 6-\mathrm{C} 5-\mathrm{H} 5$ & 119.3 & $\mathrm{C} 14-\mathrm{C} 13-\mathrm{H} 13$ & 119.6 \\
\hline $\mathrm{C} 4-\mathrm{C} 5-\mathrm{H} 5$ & 119.3 & $\mathrm{C} 12-\mathrm{C} 13-\mathrm{H} 13$ & 119.6 \\
\hline $\mathrm{N} 1-\mathrm{C} 6-\mathrm{C} 5$ & $120.9(8)$ & $\mathrm{C} 13-\mathrm{C} 14-\mathrm{C} 9$ & $120.6(10)$ \\
\hline $\mathrm{N} 1-\mathrm{C} 6-\mathrm{H} 6$ & 119.6 & $\mathrm{C} 13-\mathrm{C} 14-\mathrm{H} 14$ & 119.7 \\
\hline $\mathrm{C} 5-\mathrm{C} 6-\mathrm{H} 6$ & 119.6 & C9-C14-H14 & 119.7 \\
\hline $\mathrm{C} 6-\mathrm{N} 1-\mathrm{C} 2-\mathrm{C} 3$ & $0.4(14)$ & $\mathrm{C} 3-\mathrm{C} 4-\mathrm{C} 7-\mathrm{N} 2$ & $18.1(13)$ \\
\hline $\mathrm{C} 1-\mathrm{N} 1-\mathrm{C} 2-\mathrm{C} 3$ & $179.7(9)$ & $\mathrm{C} 5-\mathrm{C} 4-\mathrm{C} 7-\mathrm{N} 2$ & $-164.0(9)$ \\
\hline $\mathrm{N} 1-\mathrm{C} 2-\mathrm{C} 3-\mathrm{C} 4$ & $-2.5(14)$ & $\mathrm{C} 7-\mathrm{N} 2-\mathrm{C} 8-\mathrm{C} 9$ & $-75.0(11)$ \\
\hline $\mathrm{C} 2-\mathrm{C} 3-\mathrm{C} 4-\mathrm{C} 5$ & $3.5(13)$ & $\mathrm{N} 2-\mathrm{C} 8-\mathrm{C} 9-\mathrm{C} 14$ & $104.6(9)$ \\
\hline $\mathrm{C} 2-\mathrm{C} 3-\mathrm{C} 4-\mathrm{C} 7$ & $-178.6(9)$ & $\mathrm{N} 2-\mathrm{C} 8-\mathrm{C} 9-\mathrm{C} 10$ & $-77.6(11)$ \\
\hline $\mathrm{C} 3-\mathrm{C} 4-\mathrm{C} 5-\mathrm{C} 6$ & $-2.7(14)$ & $\mathrm{C} 14-\mathrm{C} 9-\mathrm{C} 10-\mathrm{C} 11$ & $-4.3(13)$ \\
\hline $\mathrm{C} 7-\mathrm{C} 4-\mathrm{C} 5-\mathrm{C} 6$ & $179.3(9)$ & $\mathrm{C} 8-\mathrm{C} 9-\mathrm{C} 10-\mathrm{C} 11$ & $177.8(9)$ \\
\hline $\mathrm{C} 2-\mathrm{N} 1-\mathrm{C} 6-\mathrm{C} 5$ & $0.5(14)$ & $\mathrm{C} 9-\mathrm{C} 10-\mathrm{C} 11-\mathrm{C} 12$ & $3.4(15)$ \\
\hline $\mathrm{C} 1-\mathrm{N} 1-\mathrm{C} 6-\mathrm{C} 5$ & $-178.9(9)$ & $\mathrm{C} 10-\mathrm{C} 11-\mathrm{C} 12-\mathrm{C} 13$ & $-0.5(15)$ \\
\hline $\mathrm{C} 4-\mathrm{C} 5-\mathrm{C} 6-\mathrm{N} 1$ & $0.7(16)$ & $\mathrm{C} 11-\mathrm{C} 12-\mathrm{C} 13-\mathrm{C} 14$ & $-1.3(15)$ \\
\hline $\mathrm{C} 8-\mathrm{N} 2-\mathrm{C} 7-\mathrm{O} 1$ & $2.3(14)$ & $\mathrm{C} 12-\mathrm{C} 13-\mathrm{C} 14-\mathrm{C} 9$ & $0.2(15)$ \\
\hline $\mathrm{C} 8-\mathrm{N} 2-\mathrm{C} 7-\mathrm{C} 4$ & $-176.2(8)$ & $\mathrm{C} 10-\mathrm{C} 9-\mathrm{C} 14-\mathrm{C} 13$ & $2.6(13)$ \\
\hline $\mathrm{C} 3-\mathrm{C} 4-\mathrm{C} 7-\mathrm{O} 1$ & $-160.5(9)$ & $\mathrm{C} 8-\mathrm{C} 9-\mathrm{C} 14-\mathrm{C} 13$ & $-179.5(9)$ \\
\hline $\mathrm{C} 5-\mathrm{C} 4-\mathrm{C} 7-\mathrm{O} 1$ & $17.4(13)$ & & \\
\hline
\end{tabular}

Symmetry code: (i) $-x+1,-y,-z+1$.

Hydrogen-bond geometry $\left(\AA,{ }^{\circ}\right)$

\begin{tabular}{lllll}
\hline$D-\mathrm{H} \cdots A$ & $D-\mathrm{H}$ & $\mathrm{H} \cdots A$ & $D \cdots A$ & $D-\mathrm{H} \cdots A$ \\
\hline $\mathrm{N} 2-\mathrm{H} 2 \cdots \mathrm{I} 2$ & 0.86 & 2.84 & $3.632(7)$ & 154 \\
$\mathrm{C} 2-\mathrm{H} 2 A \cdots \mathrm{I} 2^{\mathrm{ii}}$ & 0.93 & 3.18 & $4.053(9)$ & 158 \\
$\mathrm{C} 1-\mathrm{H} 1 B \cdots \mathrm{I} 2^{\mathrm{ii}}$ & 0.96 & 3.11 & $3.992(9)$ & 153 \\
$\mathrm{C} 1-\mathrm{H} 1 C \cdots \mathrm{I} 2^{\mathrm{iii}}$ & 0.96 & 2.96 & $3.908(9)$ & 171 \\
$\mathrm{C} 1-\mathrm{H} 1 A \cdots \mathrm{I} 1^{\text {iv }}$ & 0.96 & 3.00 & $3.824(10)$ & 145 \\
$\mathrm{C} 5-\mathrm{H} 5 \cdots \mathrm{O} 1^{\mathrm{v}}$ & 0.93 & 2.59 & $3.328(11)$ & 136 \\
$\mathrm{C} 8-\mathrm{H} 8 B \cdots \mathrm{C} 11^{\text {vi }}$ & 0.97 & 2.80 & $3.590(15)$ & 140 \\
$\mathrm{C} 8-\mathrm{H} 8 B \cdots \mathrm{C} 10^{\text {vi }}$ & 0.97 & 2.76 & $3.694(14)$ & 162
\end{tabular}

Symmetry codes: (ii) $-x+1,-y+1,-z+1$; (iii) $x-1 / 2,-y+3 / 2, z-1 / 2$; (iv) $x-1 / 2,-y+1 / 2, z-1 / 2$; (v) $-x,-y+1,-z+1$; (vi) $-x+1 / 2, y-1 / 2,-z+3 / 2$. 
4-[(Benzylamino)carbonyl]-1-methylpyridinium triiodide (II)

Crystal data

$\mathrm{C}_{14} \mathrm{H}_{15} \mathrm{~N}_{2} \mathrm{O}^{+} \cdot \mathrm{I}_{3}^{-}$

$M_{r}=608.61$

Monoclinic, $P 2_{1} / c$

$a=9.914(2) \AA$

$b=27.805$ (4) $\AA$

$c=14.113(3) \AA$

$\beta=107.83(2)^{\circ}$

$V=3703.4(12) \AA^{3}$

$Z=8$

\section{Data collection}

Xcalibur, Sapphire3 diffractometer

Radiation source: Enhance (Mo) X-ray Source

Detector resolution: 16.1827 pixels $\mathrm{mm}^{-1}$

$\omega$ scans

Absorption correction: multi-scan

(CrysAlisPro; Rigaku OD, 2018)

$T_{\min }=0.347, T_{\max }=1.000$

\section{Refinement}

Refinement on $F^{2}$

Least-squares matrix: full

$R\left[F^{2}>2 \sigma\left(F^{2}\right)\right]=0.065$

$w R\left(F^{2}\right)=0.187$

$S=0.97$

6496 reflections

371 parameters

0 restraints
$F(000)=2242$

$D_{\mathrm{x}}=2.183 \mathrm{Mg} \mathrm{m}^{-3}$

Mo $K \alpha$ radiation, $\lambda=0.71073 \AA$

Cell parameters from 1078 reflections

$\theta=3.1-18.1^{\circ}$

$\mu=5.07 \mathrm{~mm}^{-1}$

$T=293 \mathrm{~K}$

Block, yellow

$0.03 \times 0.03 \times 0.02 \mathrm{~mm}$

21040 measured reflections

6496 independent reflections

2548 reflections with $I>2 \sigma(I)$

$R_{\text {int }}=0.124$

$\theta_{\text {max }}=25.0^{\circ}, \theta_{\text {min }}=3.0^{\circ}$

$h=-8 \rightarrow 11$

$k=-33 \rightarrow 33$

$l=-16 \rightarrow 16$

Hydrogen site location: inferred from neighbouring sites

$\mathrm{H}$-atom parameters constrained

$w=1 /\left[\sigma^{2}\left(F_{\mathrm{o}}^{2}\right)+(0.0424 P)^{2}\right]$

where $P=\left(F_{\mathrm{o}}^{2}+2 F_{\mathrm{c}}{ }^{2}\right) / 3$

$(\Delta / \sigma)_{\max }<0.001$

$\Delta \rho_{\max }=0.70 \mathrm{e} \AA^{-3}$

$\Delta \rho_{\min }=-0.77$ e $\AA^{-3}$

Special details

Geometry. All esds (except the esd in the dihedral angle between two 1.s. planes) are estimated using the full covariance matrix. The cell esds are taken into account individually in the estimation of esds in distances, angles and torsion angles; correlations between esds in cell parameters are only used when they are defined by crystal symmetry. An approximate (isotropic) treatment of cell esds is used for estimating esds involving l.s. planes.

Fractional atomic coordinates and isotropic or equivalent isotropic displacement parameters $\left(\AA^{2}\right)$

\begin{tabular}{llllll}
\hline & $x$ & $y$ & $z$ & $U_{\text {iso }} * / U_{\text {eq }}$ & Occ. $(<1)$ \\
\hline I1 & $0.45921(12)$ & $0.79328(4)$ & $0.65364(9)$ & $0.0868(4)$ & \\
I2 & $0.46503(14)$ & $0.71598(5)$ & $0.78702(11)$ & $0.1072(4)$ & \\
I3 & $0.45434(15)$ & $0.87375(4)$ & $0.50883(10)$ & $0.1061(5)$ & \\
I4 & 0.000000 & 1.000000 & 0.500000 & $0.1048(6)$ & \\
I5 & $-0.09620(18)$ & $0.93095(5)$ & $0.62111(13)$ & $0.1313(6)$ & \\
I6 & $-0.4785(8)$ & $0.5112(2)$ & $0.5262(5)$ & $0.130(2)$ & 0.5 \\
I7 & $-0.3252(7)$ & $0.5746(2)$ & $0.6849(5)$ & $0.1504(17)$ & 0.5 \\
I7A & $-0.3531(7)$ & $0.5527(2)$ & $0.6302(5)$ & $0.1504(17)$ & 0.5 \\
O1A & $-0.1281(11)$ & $0.6399(4)$ & $0.3910(8)$ & $0.092(3)$ & \\
N1A & $0.0042(18)$ & $0.8083(4)$ & $0.3781(10)$ & $0.081(4)$ &
\end{tabular}




\begin{tabular}{|c|c|c|c|c|}
\hline $\mathrm{N} 2 \mathrm{~A}$ & $0.0997(12)$ & $0.6306(4)$ & $0.4111(9)$ & 0.078 \\
\hline $\mathrm{H} 2 \mathrm{~A}$ & 0.175586 & 0.643198 & 0.404694 & $0.094 *$ \\
\hline $\mathrm{C} 1 \mathrm{~A}$ & $0.004(2)$ & $0.8621(5)$ & $0.3785(13)$ & $0.110(6)$ \\
\hline H1AA & -0.091508 & 0.873532 & 0.349446 & $0.165^{*}$ \\
\hline $\mathrm{H} 1 \mathrm{AB}$ & 0.039744 & 0.873527 & 0.445762 & $0.165^{*}$ \\
\hline $\mathrm{H} 1 \mathrm{AC}$ & 0.062284 & 0.873811 & 0.340557 & $0.165^{*}$ \\
\hline $\mathrm{C} 2 \mathrm{~A}$ & 0.1245 (19) & $0.7843(6)$ & $0.4148(13)$ & $0.096(5)$ \\
\hline $\mathrm{H} 2 \mathrm{AA}$ & 0.209343 & 0.801051 & 0.438759 & $0.115^{*}$ \\
\hline C3A & $0.1251(16)$ & $0.7345(6)$ & $0.4178(12)$ & $0.088(5)$ \\
\hline $\mathrm{H} 3 \mathrm{~A}$ & 0.210205 & 0.717786 & 0.440984 & $0.106^{*}$ \\
\hline $\mathrm{C} 4 \mathrm{~A}$ & $0.0012(16)$ & $0.7105(6)$ & $0.3867(12)$ & 0.079 (4) \\
\hline $\mathrm{C} 5 \mathrm{~A}$ & $-0.1202(19)$ & $0.7349(6)$ & $0.3482(12)$ & $0.089(5)$ \\
\hline H5A & -0.205655 & 0.718459 & 0.324995 & $0.107^{*}$ \\
\hline C6A & $-0.1183(19)$ & $0.7837(7)$ & $0.3431(13)$ & $0.095(5)$ \\
\hline H6A & -0.202505 & 0.800379 & 0.315060 & $0.114^{*}$ \\
\hline C7A & $-0.0134(16)$ & $0.6579(6)$ & $0.3940(11)$ & 0.079 (4) \\
\hline C8A & 0.1044 (17) & $0.5796(5)$ & $0.4403(13)$ & $0.092(5)$ \\
\hline H8AA & 0.181584 & 0.564057 & 0.423495 & $0.110^{*}$ \\
\hline H8AB & 0.016959 & 0.564255 & 0.401688 & $0.110^{*}$ \\
\hline C9A & $0.1238(18)$ & $0.5715(5)$ & $0.5504(12)$ & $0.074(4)$ \\
\hline $\mathrm{C} 10 \mathrm{~A}$ & $0.252(2)$ & $0.5902(6)$ & $0.6130(16)$ & 0.097 (6) \\
\hline $\mathrm{H} 10 \mathrm{~A}$ & 0.316106 & 0.605499 & 0.587126 & $0.117^{*}$ \\
\hline $\mathrm{C} 11 \mathrm{~A}$ & $0.279(2)$ & $0.5848(6)$ & 0.7157 (17) & $0.106(6)$ \\
\hline $\mathrm{H} 11 \mathrm{~A}$ & 0.360108 & 0.597669 & 0.760162 & $0.128^{*}$ \\
\hline $\mathrm{C} 12 \mathrm{~A}$ & $0.184(2)$ & $0.5606(7)$ & $0.7493(16)$ & $0.106(6)$ \\
\hline $\mathrm{H} 12 \mathrm{~A}$ & 0.203600 & 0.554933 & 0.817130 & $0.127 *$ \\
\hline $\mathrm{C} 13 \mathrm{~A}$ & $0.060(2)$ & $0.5444(6)$ & $0.685(2)$ & $0.107(7)$ \\
\hline $\mathrm{H} 13 \mathrm{~A}$ & -0.005324 & 0.529527 & 0.710907 & $0.128 *$ \\
\hline $\mathrm{C} 14 \mathrm{~A}$ & $0.027(2)$ & $0.5491(7)$ & $0.5838(17)$ & $0.121(7)$ \\
\hline H14A & -0.057924 & 0.537576 & 0.540700 & $0.145^{*}$ \\
\hline O1B & $0.3841(10)$ & $0.6543(4)$ & $0.4242(8)$ & $0.086(3)$ \\
\hline N1B & $0.4543(16)$ & $0.5322(5)$ & $0.1922(12)$ & 0.088 (4) \\
\hline N2B & $0.6125(12)$ & $0.6628(4)$ & $0.4475(8)$ & $0.076(4)$ \\
\hline $\mathrm{H} 2 \mathrm{~B}$ & 0.684595 & 0.655003 & 0.429131 & $0.091 *$ \\
\hline C1B & 0.4388 (19) & $0.4928(6)$ & 0.1164 (14) & $0.108(6)$ \\
\hline H1BA & 0.530747 & 0.480708 & 0.119642 & $0.162 *$ \\
\hline H1BB & 0.382401 & 0.467282 & 0.130134 & $0.162 *$ \\
\hline $\mathrm{H} 1 \mathrm{BC}$ & 0.393375 & 0.505462 & 0.051088 & $0.162 *$ \\
\hline C2B & $0.581(2)$ & $0.5433(6)$ & $0.2527(16)$ & $0.102(6)$ \\
\hline $\mathrm{H} 2 \mathrm{BA}$ & 0.658994 & 0.525293 & 0.250589 & $0.123^{*}$ \\
\hline $\mathrm{C} 3 \mathrm{~B}$ & $0.5997(15)$ & $0.5805(5)$ & $0.3180(12)$ & $0.074(4)$ \\
\hline H3B & 0.690985 & 0.589253 & 0.355599 & $0.088 *$ \\
\hline C4B & $0.4836(14)$ & $0.6059(5)$ & $0.3296(11)$ & 0.069 (4) \\
\hline C5B & $0.356(2)$ & $0.5904(6)$ & $0.2686(12)$ & $0.089(5)$ \\
\hline H5B & 0.273994 & 0.605203 & 0.272963 & $0.107^{*}$ \\
\hline C6B & $0.3436(18)$ & $0.5548(6)$ & $0.2030(12)$ & $0.090(5)$ \\
\hline H6B & 0.253572 & 0.545749 & 0.163528 & $0.107^{*}$ \\
\hline C7B & $0.4904(17)$ & $0.6428(5)$ & $0.4034(11)$ & $0.071(4)$ \\
\hline
\end{tabular}




\begin{tabular}{lllll} 
C8B & $0.6312(18)$ & $0.6988(5)$ & $0.5285(12)$ & $0.085(5)$ \\
H8BA & 0.569928 & 0.726128 & 0.502782 & $0.102^{*}$ \\
H8BB & 0.601106 & 0.684595 & 0.581491 & $0.102^{*}$ \\
C9B & $0.7765(16)$ & $0.7161(6)$ & $0.5702(11)$ & $0.072(4)$ \\
C10B & $0.8890(17)$ & $0.6853(6)$ & $0.6049(12)$ & $0.084(4)$ \\
H10B & 0.872179 & 0.652312 & 0.601274 & $0.100^{*}$ \\
C11B & $1.024(2)$ & $0.7013(7)$ & $0.6443(13)$ & $0.097(5)$ \\
H11B & 1.097443 & 0.679375 & 0.668821 & $0.116^{*}$ \\
C12B & $1.0525(19)$ & $0.7495(8)$ & $0.6480(11)$ & $0.094(5)$ \\
H12B & 1.144660 & 0.760430 & 0.676679 & $0.112^{*}$ \\
C13B & $0.946(2)$ & $0.7812(7)$ & $0.6098(13)$ & $0.096(5)$ \\
H13B & 0.966127 & 0.813824 & 0.608168 & $0.115^{*}$ \\
C14B & $0.8040(16)$ & $0.7646(6)$ & $0.5720(11)$ & $0.081(5)$ \\
H14B & 0.730116 & 0.786363 & 0.548735 & $0.097^{*}$ \\
\hline
\end{tabular}

Atomic displacement parameters $\left(\AA^{2}\right)$

\begin{tabular}{|c|c|c|c|c|c|c|}
\hline & $U^{11}$ & $U^{22}$ & $U^{33}$ & $U^{12}$ & $U^{13}$ & $U^{23}$ \\
\hline I1 & $0.0752(7)$ & $0.0882(8)$ & $0.0963(8)$ & $-0.0041(6)$ & $0.0254(6)$ & $-0.0169(6)$ \\
\hline $\mathrm{I} 2$ & $0.0868(9)$ & $0.0985(9)$ & $0.1316(11)$ & $-0.0025(7)$ & $0.0264(8)$ & $0.0075(8)$ \\
\hline I3 & $0.1210(11)$ & $0.0961(9)$ & $0.0995(9)$ & $-0.0168(8)$ & $0.0312(8)$ & $-0.0034(7)$ \\
\hline I4 & $0.0852(12)$ & $0.0960(13)$ & 0.1177 (14) & $0.0118(10)$ & $0.0082(10)$ & $-0.0004(10)$ \\
\hline I5 & $0.1384(14)$ & 0.1108 (11) & $0.1504(14)$ & $0.0019(10)$ & $0.0526(12)$ & $0.0023(9)$ \\
\hline I6 & $0.099(4)$ & $0.131(5)$ & $0.180(7)$ & $0.027(3)$ & $0.072(5)$ & 0.077 (4) \\
\hline I7 & $0.117(3)$ & $0.148(4)$ & $0.200(6)$ & $-0.001(3)$ & $0.069(4)$ & $0.048(3)$ \\
\hline I7A & $0.117(3)$ & $0.148(4)$ & $0.200(6)$ & $-0.001(3)$ & 0.069 (4) & $0.048(3)$ \\
\hline O1A & $0.068(7)$ & $0.091(8)$ & $0.123(10)$ & $-0.009(6)$ & $0.039(7)$ & $-0.023(6)$ \\
\hline N1A & $0.106(11)$ & $0.062(8)$ & $0.085(9)$ & $0.004(8)$ & $0.043(9)$ & $0.012(7)$ \\
\hline $\mathrm{N} 2 \mathrm{~A}$ & $0.048(7)$ & $0.080(9)$ & $0.105(10)$ & $-0.015(7)$ & $0.022(7)$ & $-0.018(7)$ \\
\hline $\mathrm{C} 1 \mathrm{~A}$ & $0.137(18)$ & $0.073(11)$ & $0.116(15)$ & $-0.004(11)$ & $0.031(13)$ & $0.013(10)$ \\
\hline $\mathrm{C} 2 \mathrm{~A}$ & $0.071(12)$ & $0.091(13)$ & $0.124(15)$ & $-0.013(10)$ & $0.029(11)$ & $0.002(11)$ \\
\hline $\mathrm{C} 3 \mathrm{~A}$ & $0.058(10)$ & 0.079 (11) & 0.115 (14) & $-0.002(9)$ & $0.008(9)$ & $0.013(9)$ \\
\hline $\mathrm{C} 4 \mathrm{~A}$ & $0.048(9)$ & $0.093(12)$ & $0.086(11)$ & $-0.011(9)$ & $0.005(8)$ & $-0.001(9)$ \\
\hline C5A & 0.085 (13) & 0.089 (13) & 0.087 (12) & $-0.024(11)$ & 0.018 (10) & $-0.002(9)$ \\
\hline C6A & 0.069 (11) & $0.121(16)$ & $0.098(13)$ & 0.017 (12) & $0.031(10)$ & $0.017(11)$ \\
\hline C7A & $0.050(9)$ & $0.110(14)$ & $0.076(11)$ & $-0.004(10)$ & $0.021(8)$ & $-0.010(9)$ \\
\hline C8A & $0.078(12)$ & 0.075 (11) & $0.125(16)$ & $-0.011(9)$ & 0.035 (11) & $-0.017(10)$ \\
\hline C9A & $0.080(11)$ & $0.064(10)$ & 0.067 (11) & $-0.006(8)$ & $0.009(9)$ & $-0.018(8)$ \\
\hline C10A & $0.108(15)$ & $0.081(12)$ & $0.122(16)$ & $-0.001(11)$ & 0.063 (14) & $-0.017(11)$ \\
\hline C11A & 0.087 (14) & $0.105(15)$ & 0.125 (18) & $-0.001(11)$ & 0.029 (13) & $-0.021(12)$ \\
\hline C12A & $0.094(15)$ & $0.113(16)$ & $0.112(16)$ & $0.024(13)$ & 0.034 (14) & $0.002(12)$ \\
\hline C13A & $0.114(17)$ & $0.074(12)$ & $0.16(2)$ & $0.003(12)$ & 0.084 (17) & $0.024(13)$ \\
\hline C14A & $0.125(18)$ & $0.140(18)$ & 0.120 (19) & $-0.011(15)$ & $0.072(16)$ & $-0.011(14)$ \\
\hline O1B & $0.058(7)$ & $0.107(8)$ & $0.098(8)$ & $-0.007(6)$ & $0.029(6)$ & $-0.016(6)$ \\
\hline N1B & $0.087(10)$ & $0.073(9)$ & $0.120(12)$ & 0.009 (8) & $0.058(10)$ & $-0.002(8)$ \\
\hline $\mathrm{N} 2 \mathrm{~B}$ & $0.042(7)$ & $0.105(10)$ & $0.078(9)$ & $-0.011(7)$ & $0.014(6)$ & $-0.018(7)$ \\
\hline C1B & $0.105(15)$ & $0.101(13)$ & $0.120(15)$ & $-0.017(11)$ & $0.036(13)$ & $-0.017(12)$ \\
\hline $\mathrm{C} 2 \mathrm{~B}$ & $0.075(13)$ & $0.060(11)$ & $0.18(2)$ & $-0.006(10)$ & $0.048(14)$ & $0.007(12)$ \\
\hline
\end{tabular}




$\begin{array}{lllllll} & & & & \\ \text { C3B } & 0.056(9) & 0.054(9) & 0.109(13) & -0.012(7) & 0.021(9) & -0.012(8) \\ \text { C4B } & 0.043(8) & 0.073(10) & 0.086(11) & -0.010(7) & 0.011(7) & -0.005(8) \\ \text { C5B } & 0.113(15) & 0.084(12) & 0.085(12) & 0.011(11) & 0.054(12) & -0.009(9) \\ \text { C6B } & 0.072(11) & 0.116(15) & 0.081(12) & -0.019(11) & 0.023(10) & -0.007(10) \\ \text { C7B } & 0.073(10) & 0.075(10) & 0.077(11) & 0.015(9) & 0.038(9) & 0.005(8) \\ \text { C8B } & 0.094(13) & 0.075(10) & 0.086(11) & -0.011(9) & 0.028(10) & -0.014(9) \\ \text { C9B } & 0.068(10) & 0.077(11) & 0.075(10) & -0.012(9) & 0.030(8) & -0.018(8) \\ \text { C10B } & 0.066(11) & 0.089(12) & 0.089(12) & 0.011(10) & 0.014(9) & 0.003(9) \\ \text { C11B } & 0.077(13) & 0.125(16) & 0.092(13) & 0.005(12) & 0.030(11) & 0.003(11) \\ \text { C12B } & 0.072(12) & 0.140(17) & 0.064(11) & -0.021(13) & 0.014(9) & -0.016(11) \\ \text { C13B } & 0.090(13) & 0.104(13) & 0.097(13) & -0.006(12) & 0.034(11) & 0.002(11) \\ \text { C14B } & 0.052(9) & 0.112(14) & 0.069(10) & 0.014(9) & 0.005(8) & -0.014(9) \\ & & & & & \end{array}$

Geometric parameters $\left(\AA,{ }^{\circ}\right)$

\begin{tabular}{|c|c|c|c|}
\hline $\mathrm{I} 1-\mathrm{I} 2$ & $2.8459(18)$ & $\mathrm{C} 12 \mathrm{~A}-\mathrm{H} 12 \mathrm{~A}$ & 0.9300 \\
\hline $\mathrm{I} 1-\mathrm{I} 3$ & $3.0206(17)$ & $\mathrm{C} 13 \mathrm{~A}-\mathrm{C} 14 \mathrm{~A}$ & $1.37(3)$ \\
\hline I4-I5 & $2.9181(15)$ & $\mathrm{C} 13 \mathrm{~A}-\mathrm{H} 13 \mathrm{~A}$ & 0.9300 \\
\hline $\mathrm{I} 4-\mathrm{I} 5^{\mathrm{i}}$ & $2.9181(15)$ & $\mathrm{C} 14 \mathrm{~A}-\mathrm{H} 14 \mathrm{~A}$ & 0.9300 \\
\hline $\mathrm{I} 6-\mathrm{I} 6^{\mathrm{ii}}$ & $0.962(9)$ & $\mathrm{O} 1 \mathrm{~B}-\mathrm{C} 7 \mathrm{~B}$ & $1.220(15)$ \\
\hline I6-I7A & $1.977(7)$ & $\mathrm{N} 1 \mathrm{~B}-\mathrm{C} 6 \mathrm{~B}$ & $1.313(18)$ \\
\hline I6-I7 & $2.890(7)$ & $\mathrm{N} 1 \mathrm{~B}-\mathrm{C} 2 \mathrm{~B}$ & $1.32(2)$ \\
\hline I6-I7 $\mathrm{A}^{\mathrm{ii}}$ & $2.925(7)$ & $\mathrm{N} 1 \mathrm{~B}-\mathrm{C} 1 \mathrm{~B}$ & $1.504(19)$ \\
\hline I7-I7A & $0.957(7)$ & $\mathrm{N} 2 \mathrm{~B}-\mathrm{C} 7 \mathrm{~B}$ & 1.305 (17) \\
\hline $\mathrm{O} 1 \mathrm{~A}-\mathrm{C} 7 \mathrm{~A}$ & $1.231(16)$ & $\mathrm{N} 2 \mathrm{~B}-\mathrm{C} 8 \mathrm{~B}$ & $1.488(17)$ \\
\hline $\mathrm{N} 1 \mathrm{~A}-\mathrm{C} 2 \mathrm{~A}$ & 1.327 (19) & $\mathrm{N} 2 \mathrm{~B}-\mathrm{H} 2 \mathrm{~B}$ & 0.8600 \\
\hline $\mathrm{N} 1 \mathrm{~A}-\mathrm{C} 6 \mathrm{~A}$ & $1.35(2)$ & $\mathrm{C} 1 \mathrm{~B}-\mathrm{H} 1 \mathrm{BA}$ & 0.9600 \\
\hline $\mathrm{N} 1 \mathrm{~A}-\mathrm{C} 1 \mathrm{~A}$ & 1.495 (17) & $\mathrm{C} 1 \mathrm{~B}-\mathrm{H} 1 \mathrm{BB}$ & 0.9600 \\
\hline $\mathrm{N} 2 \mathrm{~A}-\mathrm{C} 7 \mathrm{~A}$ & $1.315(17)$ & $\mathrm{C} 1 \mathrm{~B}-\mathrm{H} 1 \mathrm{BC}$ & 0.9600 \\
\hline $\mathrm{N} 2 \mathrm{~A}-\mathrm{C} 8 \mathrm{~A}$ & $1.472(17)$ & $\mathrm{C} 2 \mathrm{~B}-\mathrm{C} 3 \mathrm{~B}$ & $1.36(2)$ \\
\hline $\mathrm{N} 2 \mathrm{~A}-\mathrm{H} 2 \mathrm{~A}$ & 0.8600 & $\mathrm{C} 2 \mathrm{~B}-\mathrm{H} 2 \mathrm{BA}$ & 0.9300 \\
\hline $\mathrm{C} 1 \mathrm{~A}-\mathrm{H} 1 \mathrm{AA}$ & 0.9600 & $\mathrm{C} 3 \mathrm{~B}-\mathrm{C} 4 \mathrm{~B}$ & $1.403(18)$ \\
\hline $\mathrm{C} 1 \mathrm{~A}-\mathrm{H} 1 \mathrm{AB}$ & 0.9600 & $\mathrm{C} 3 \mathrm{~B}-\mathrm{H} 3 \mathrm{~B}$ & 0.9300 \\
\hline $\mathrm{C} 1 \mathrm{~A}-\mathrm{H} 1 \mathrm{AC}$ & 0.9600 & $\mathrm{C} 4 \mathrm{~B}-\mathrm{C} 5 \mathrm{~B}$ & $1.36(2)$ \\
\hline $\mathrm{C} 2 \mathrm{~A}-\mathrm{C} 3 \mathrm{~A}$ & $1.39(2)$ & $\mathrm{C} 4 \mathrm{~B}-\mathrm{C} 7 \mathrm{~B}$ & 1.447 (19) \\
\hline $\mathrm{C} 2 \mathrm{~A}-\mathrm{H} 2 \mathrm{AA}$ & 0.9300 & $\mathrm{C} 5 \mathrm{~B}-\mathrm{C} 6 \mathrm{~B}$ & $1.335(19)$ \\
\hline $\mathrm{C} 3 \mathrm{~A}-\mathrm{C} 4 \mathrm{~A}$ & $1.348(19)$ & $\mathrm{C} 5 \mathrm{~B}-\mathrm{H} 5 \mathrm{~B}$ & 0.9300 \\
\hline $\mathrm{C} 3 \mathrm{~A}-\mathrm{H} 3 \mathrm{~A}$ & 0.9300 & $\mathrm{C} 6 \mathrm{~B}-\mathrm{H} 6 \mathrm{~B}$ & 0.9300 \\
\hline $\mathrm{C} 4 \mathrm{~A}-\mathrm{C} 5 \mathrm{~A}$ & $1.34(2)$ & $\mathrm{C} 8 \mathrm{~B}-\mathrm{C} 9 \mathrm{~B}$ & $1.46(2)$ \\
\hline $\mathrm{C} 4 \mathrm{~A}-\mathrm{C} 7 \mathrm{~A}$ & $1.48(2)$ & C8B-H8BA & 0.9700 \\
\hline $\mathrm{C} 5 \mathrm{~A}-\mathrm{C} 6 \mathrm{~A}$ & $1.36(2)$ & $\mathrm{C} 8 \mathrm{~B}-\mathrm{H} 8 \mathrm{BB}$ & 0.9700 \\
\hline $\mathrm{C} 5 \mathrm{~A}-\mathrm{H} 5 \mathrm{~A}$ & 0.9300 & $\mathrm{C} 9 \mathrm{~B}-\mathrm{C} 10 \mathrm{~B}$ & $1.372(19)$ \\
\hline $\mathrm{C} 6 \mathrm{~A}-\mathrm{H} 6 \mathrm{~A}$ & 0.9300 & $\mathrm{C} 9 \mathrm{~B}-\mathrm{C} 14 \mathrm{~B}$ & $1.374(19)$ \\
\hline $\mathrm{C} 8 \mathrm{~A}-\mathrm{C} 9 \mathrm{~A}$ & $1.52(2)$ & $\mathrm{C} 10 \mathrm{~B}-\mathrm{C} 11 \mathrm{~B}$ & $1.36(2)$ \\
\hline $\mathrm{C} 8 \mathrm{~A}-\mathrm{H} 8 \mathrm{AA}$ & 0.9700 & $\mathrm{C} 10 \mathrm{~B}-\mathrm{H} 10 \mathrm{~B}$ & 0.9300 \\
\hline $\mathrm{C} 8 \mathrm{~A}-\mathrm{H} 8 \mathrm{AB}$ & 0.9700 & $\mathrm{C} 11 \mathrm{~B}-\mathrm{C} 12 \mathrm{~B}$ & $1.37(2)$ \\
\hline $\mathrm{C} 9 \mathrm{~A}-\mathrm{C} 14 \mathrm{~A}$ & $1.35(2)$ & $\mathrm{C} 11 \mathrm{~B}-\mathrm{H} 11 \mathrm{~B}$ & 0.9300 \\
\hline C9A-C10A & $1.40(2)$ & $\mathrm{C} 12 \mathrm{~B}-\mathrm{C} 13 \mathrm{~B}$ & $1.35(2)$ \\
\hline
\end{tabular}




\begin{tabular}{|c|c|c|c|}
\hline $\mathrm{C} 10 \mathrm{~A}-\mathrm{C} 11 \mathrm{~A}$ & $1.40(2)$ & $\mathrm{C} 12 \mathrm{~B}-\mathrm{H} 12 \mathrm{~B}$ & 0.9300 \\
\hline $\mathrm{C} 10 \mathrm{~A}-\mathrm{H} 10 \mathrm{~A}$ & 0.9300 & $\mathrm{C} 13 \mathrm{~B}-\mathrm{C} 14 \mathrm{~B}$ & $1.42(2)$ \\
\hline $\mathrm{C} 11 \mathrm{~A}-\mathrm{C} 12 \mathrm{~A}$ & $1.35(2)$ & $\mathrm{C} 13 \mathrm{~B}-\mathrm{H} 13 \mathrm{~B}$ & 0.9300 \\
\hline $\mathrm{C} 11 \mathrm{~A}-\mathrm{H} 11 \mathrm{~A}$ & 0.9300 & $\mathrm{C} 14 \mathrm{~B}-\mathrm{H} 14 \mathrm{~B}$ & 0.9300 \\
\hline $\mathrm{C} 12 \mathrm{~A}-\mathrm{C} 13 \mathrm{~A}$ & $1.36(3)$ & & \\
\hline $\mathrm{I} 2-\mathrm{I} 1-\mathrm{I} 3$ & $178.72(5)$ & $\mathrm{C} 12 \mathrm{~A}-\mathrm{C} 13 \mathrm{~A}-\mathrm{C} 14 \mathrm{~A}$ & $122.8(18)$ \\
\hline $\mathrm{I} 5-\mathrm{I} 4-\mathrm{I} 5^{\mathrm{i}}$ & 180.0 & $\mathrm{C} 12 \mathrm{~A}-\mathrm{C} 13 \mathrm{~A}-\mathrm{H} 13 \mathrm{~A}$ & 118.6 \\
\hline $\mathrm{I} 6^{\mathrm{ii}}-\mathrm{I} 6-\mathrm{I} 7 \mathrm{~A}$ & $168.1(11)$ & $\mathrm{C} 14 \mathrm{~A}-\mathrm{C} 13 \mathrm{~A}-\mathrm{H} 13 \mathrm{~A}$ & 118.6 \\
\hline $\mathrm{I} 6^{\mathrm{ii}-\mathrm{I} 6-\mathrm{I} 7}$ & $174.9(11)$ & $\mathrm{C} 9 \mathrm{~A}-\mathrm{C} 14 \mathrm{~A}-\mathrm{C} 13 \mathrm{~A}$ & $116(2)$ \\
\hline $\mathrm{I} 7 \mathrm{~A}-\mathrm{I} 6-\mathrm{I} 7$ & $6.9(3)$ & $\mathrm{C} 9 \mathrm{~A}-\mathrm{C} 14 \mathrm{~A}-\mathrm{H} 14 \mathrm{~A}$ & 121.9 \\
\hline $\mathrm{I}^{\mathrm{ii}}-\mathrm{I} 6-\mathrm{I} 7 \mathrm{~A}^{\mathrm{ii}}$ & $8.0(8)$ & $\mathrm{C} 13 \mathrm{~A}-\mathrm{C} 14 \mathrm{~A}-\mathrm{H} 14 \mathrm{~A}$ & 121.9 \\
\hline $\mathrm{I} 7 \mathrm{~A}-\mathrm{I} 6-\mathrm{I} 7 \mathrm{~A}^{\mathrm{ii}}$ & $176.1(4)$ & $\mathrm{C} 6 \mathrm{~B}-\mathrm{N} 1 \mathrm{~B}-\mathrm{C} 2 \mathrm{~B}$ & $118.4(15)$ \\
\hline I7-I6-I7A $\mathrm{A}^{\mathrm{ii}}$ & $176.9(4)$ & $\mathrm{C} 6 \mathrm{~B}-\mathrm{N} 1 \mathrm{~B}-\mathrm{C} 1 \mathrm{~B}$ & $121.6(16)$ \\
\hline $\mathrm{I} 7 \mathrm{~A}-\mathrm{I} 7-\mathrm{I} 6$ & $14.4(7)$ & $\mathrm{C} 2 \mathrm{~B}-\mathrm{N} 1 \mathrm{~B}-\mathrm{C} 1 \mathrm{~B}$ & $120.0(15)$ \\
\hline $\mathrm{I} 7-\mathrm{I} 7 \mathrm{~A}-\mathrm{I} 6$ & $158.7(10)$ & $\mathrm{C} 7 \mathrm{~B}-\mathrm{N} 2 \mathrm{~B}-\mathrm{C} 8 \mathrm{~B}$ & $122.2(12)$ \\
\hline $\mathrm{I} 7-\mathrm{I} 7 \mathrm{~A}-\mathrm{I} 6^{\mathrm{ii}}$ & $162.5(9)$ & $\mathrm{C} 7 \mathrm{~B}-\mathrm{N} 2 \mathrm{~B}-\mathrm{H} 2 \mathrm{~B}$ & 118.9 \\
\hline $\mathrm{I} 6-\mathrm{I} 7 \mathrm{~A}-\mathrm{I} 6^{\mathrm{ii}}$ & $3.9(4)$ & $\mathrm{C} 8 \mathrm{~B}-\mathrm{N} 2 \mathrm{~B}-\mathrm{H} 2 \mathrm{~B}$ & 118.9 \\
\hline $\mathrm{C} 2 \mathrm{~A}-\mathrm{N} 1 \mathrm{~A}-\mathrm{C} 6 \mathrm{~A}$ & $119.2(14)$ & $\mathrm{N} 1 \mathrm{~B}-\mathrm{C} 1 \mathrm{~B}-\mathrm{H} 1 \mathrm{BA}$ & 109.5 \\
\hline $\mathrm{C} 2 \mathrm{~A}-\mathrm{N} 1 \mathrm{~A}-\mathrm{C} 1 \mathrm{~A}$ & $120.4(16)$ & $\mathrm{N} 1 \mathrm{~B}-\mathrm{C} 1 \mathrm{~B}-\mathrm{H} 1 \mathrm{BB}$ & 109.5 \\
\hline $\mathrm{C} 6 \mathrm{~A}-\mathrm{N} 1 \mathrm{~A}-\mathrm{C} 1 \mathrm{~A}$ & $120.3(16)$ & $\mathrm{H} 1 \mathrm{BA}-\mathrm{C} 1 \mathrm{~B}-\mathrm{H} 1 \mathrm{BB}$ & 109.5 \\
\hline $\mathrm{C} 7 \mathrm{~A}-\mathrm{N} 2 \mathrm{~A}-\mathrm{C} 8 \mathrm{~A}$ & $124.0(13)$ & $\mathrm{N} 1 \mathrm{~B}-\mathrm{C} 1 \mathrm{~B}-\mathrm{H} 1 \mathrm{BC}$ & 109.5 \\
\hline $\mathrm{C} 7 \mathrm{~A}-\mathrm{N} 2 \mathrm{~A}-\mathrm{H} 2 \mathrm{~A}$ & 118.0 & $\mathrm{H} 1 \mathrm{BA}-\mathrm{C} 1 \mathrm{~B}-\mathrm{H} 1 \mathrm{BC}$ & 109.5 \\
\hline $\mathrm{C} 8 \mathrm{~A}-\mathrm{N} 2 \mathrm{~A}-\mathrm{H} 2 \mathrm{~A}$ & 118.0 & $\mathrm{H} 1 \mathrm{BB}-\mathrm{C} 1 \mathrm{~B}-\mathrm{H} 1 \mathrm{BC}$ & 109.5 \\
\hline $\mathrm{N} 1 \mathrm{~A}-\mathrm{C} 1 \mathrm{~A}-\mathrm{H} 1 \mathrm{AA}$ & 109.5 & $\mathrm{~N} 1 \mathrm{~B}-\mathrm{C} 2 \mathrm{~B}-\mathrm{C} 3 \mathrm{~B}$ & $121.5(16)$ \\
\hline $\mathrm{N} 1 \mathrm{~A}-\mathrm{C} 1 \mathrm{~A}-\mathrm{H} 1 \mathrm{AB}$ & 109.5 & $\mathrm{~N} 1 \mathrm{~B}-\mathrm{C} 2 \mathrm{~B}-\mathrm{H} 2 \mathrm{BA}$ & 119.3 \\
\hline $\mathrm{H} 1 \mathrm{AA}-\mathrm{C} 1 \mathrm{~A}-\mathrm{H} 1 \mathrm{AB}$ & 109.5 & $\mathrm{C} 3 \mathrm{~B}-\mathrm{C} 2 \mathrm{~B}-\mathrm{H} 2 \mathrm{BA}$ & 119.3 \\
\hline $\mathrm{N} 1 \mathrm{~A}-\mathrm{C} 1 \mathrm{~A}-\mathrm{H} 1 \mathrm{AC}$ & 109.5 & $\mathrm{C} 2 \mathrm{~B}-\mathrm{C} 3 \mathrm{~B}-\mathrm{C} 4 \mathrm{~B}$ & $121.0(15)$ \\
\hline $\mathrm{H} 1 \mathrm{AA}-\mathrm{C} 1 \mathrm{~A}-\mathrm{H} 1 \mathrm{AC}$ & 109.5 & $\mathrm{C} 2 \mathrm{~B}-\mathrm{C} 3 \mathrm{~B}-\mathrm{H} 3 \mathrm{~B}$ & 119.5 \\
\hline $\mathrm{H} 1 \mathrm{AB}-\mathrm{C} 1 \mathrm{~A}-\mathrm{H} 1 \mathrm{AC}$ & 109.5 & $\mathrm{C} 4 \mathrm{~B}-\mathrm{C} 3 \mathrm{~B}-\mathrm{H} 3 \mathrm{~B}$ & 119.5 \\
\hline $\mathrm{N} 1 \mathrm{~A}-\mathrm{C} 2 \mathrm{~A}-\mathrm{C} 3 \mathrm{~A}$ & $120.7(16)$ & $\mathrm{C} 5 \mathrm{~B}-\mathrm{C} 4 \mathrm{~B}-\mathrm{C} 3 \mathrm{~B}$ & $113.7(14)$ \\
\hline $\mathrm{N} 1 \mathrm{~A}-\mathrm{C} 2 \mathrm{~A}-\mathrm{H} 2 \mathrm{AA}$ & 119.6 & $\mathrm{C} 5 \mathrm{~B}-\mathrm{C} 4 \mathrm{~B}-\mathrm{C} 7 \mathrm{~B}$ & $120.6(14)$ \\
\hline $\mathrm{C} 3 \mathrm{~A}-\mathrm{C} 2 \mathrm{~A}-\mathrm{H} 2 \mathrm{AA}$ & 119.6 & $\mathrm{C} 3 \mathrm{~B}-\mathrm{C} 4 \mathrm{~B}-\mathrm{C} 7 \mathrm{~B}$ & $125.5(14)$ \\
\hline $\mathrm{C} 4 \mathrm{~A}-\mathrm{C} 3 \mathrm{~A}-\mathrm{C} 2 \mathrm{~A}$ & $119.3(16)$ & $\mathrm{C} 6 \mathrm{~B}-\mathrm{C} 5 \mathrm{~B}-\mathrm{C} 4 \mathrm{~B}$ & $122.9(16)$ \\
\hline $\mathrm{C} 4 \mathrm{~A}-\mathrm{C} 3 \mathrm{~A}-\mathrm{H} 3 \mathrm{~A}$ & 120.3 & $\mathrm{C} 6 \mathrm{~B}-\mathrm{C} 5 \mathrm{~B}-\mathrm{H} 5 \mathrm{~B}$ & 118.5 \\
\hline $\mathrm{C} 2 \mathrm{~A}-\mathrm{C} 3 \mathrm{~A}-\mathrm{H} 3 \mathrm{~A}$ & 120.3 & $\mathrm{C} 4 \mathrm{~B}-\mathrm{C} 5 \mathrm{~B}-\mathrm{H} 5 \mathrm{~B}$ & 118.5 \\
\hline $\mathrm{C} 5 \mathrm{~A}-\mathrm{C} 4 \mathrm{~A}-\mathrm{C} 3 \mathrm{~A}$ & $119.7(16)$ & $\mathrm{N} 1 \mathrm{~B}-\mathrm{C} 6 \mathrm{~B}-\mathrm{C} 5 \mathrm{~B}$ & $122.2(17)$ \\
\hline $\mathrm{C} 5 \mathrm{~A}-\mathrm{C} 4 \mathrm{~A}-\mathrm{C} 7 \mathrm{~A}$ & $115.8(14)$ & $\mathrm{N} 1 \mathrm{~B}-\mathrm{C} 6 \mathrm{~B}-\mathrm{H} 6 \mathrm{~B}$ & 118.9 \\
\hline $\mathrm{C} 3 \mathrm{~A}-\mathrm{C} 4 \mathrm{~A}-\mathrm{C} 7 \mathrm{~A}$ & $124.5(15)$ & $\mathrm{C} 5 \mathrm{~B}-\mathrm{C} 6 \mathrm{~B}-\mathrm{H} 6 \mathrm{~B}$ & 118.9 \\
\hline $\mathrm{C} 4 \mathrm{~A}-\mathrm{C} 5 \mathrm{~A}-\mathrm{C} 6 \mathrm{~A}$ & $120.1(17)$ & $\mathrm{O} 1 \mathrm{~B}-\mathrm{C} 7 \mathrm{~B}-\mathrm{N} 2 \mathrm{~B}$ & $121.1(14)$ \\
\hline $\mathrm{C} 4 \mathrm{~A}-\mathrm{C} 5 \mathrm{~A}-\mathrm{H} 5 \mathrm{~A}$ & 120.0 & $\mathrm{O} 1 \mathrm{~B}-\mathrm{C} 7 \mathrm{~B}-\mathrm{C} 4 \mathrm{~B}$ & $120.4(15)$ \\
\hline $\mathrm{C} 6 \mathrm{~A}-\mathrm{C} 5 \mathrm{~A}-\mathrm{H} 5 \mathrm{~A}$ & 120.0 & $\mathrm{~N} 2 \mathrm{~B}-\mathrm{C} 7 \mathrm{~B}-\mathrm{C} 4 \mathrm{~B}$ & $118.6(13)$ \\
\hline $\mathrm{N} 1 \mathrm{~A}-\mathrm{C} 6 \mathrm{~A}-\mathrm{C} 5 \mathrm{~A}$ & $120.8(17)$ & $\mathrm{C} 9 \mathrm{~B}-\mathrm{C} 8 \mathrm{~B}-\mathrm{N} 2 \mathrm{~B}$ & $113.9(13)$ \\
\hline $\mathrm{N} 1 \mathrm{~A}-\mathrm{C} 6 \mathrm{~A}-\mathrm{H} 6 \mathrm{~A}$ & 119.6 & $\mathrm{C} 9 \mathrm{~B}-\mathrm{C} 8 \mathrm{~B}-\mathrm{H} 8 \mathrm{BA}$ & 108.8 \\
\hline $\mathrm{C} 5 \mathrm{~A}-\mathrm{C} 6 \mathrm{~A}-\mathrm{H} 6 \mathrm{~A}$ & 119.6 & $\mathrm{~N} 2 \mathrm{~B}-\mathrm{C} 8 \mathrm{~B}-\mathrm{H} 8 \mathrm{BA}$ & 108.8 \\
\hline $\mathrm{O} 1 \mathrm{~A}-\mathrm{C} 7 \mathrm{~A}-\mathrm{N} 2 \mathrm{~A}$ & $119.8(16)$ & $\mathrm{C} 9 \mathrm{~B}-\mathrm{C} 8 \mathrm{~B}-\mathrm{H} 8 \mathrm{BB}$ & 108.8 \\
\hline $\mathrm{O} 1 \mathrm{~A}-\mathrm{C} 7 \mathrm{~A}-\mathrm{C} 4 \mathrm{~A}$ & $120.7(14)$ & $\mathrm{N} 2 \mathrm{~B}-\mathrm{C} 8 \mathrm{~B}-\mathrm{H} 8 \mathrm{BB}$ & 108.8 \\
\hline $\mathrm{N} 2 \mathrm{~A}-\mathrm{C} 7 \mathrm{~A}-\mathrm{C} 4 \mathrm{~A}$ & $119.3(14)$ & $\mathrm{H} 8 \mathrm{BA}-\mathrm{C} 8 \mathrm{~B}-\mathrm{H} 8 \mathrm{BB}$ & 107.7 \\
\hline
\end{tabular}




\begin{tabular}{|c|c|}
\hline $\mathrm{N} 2 \mathrm{~A}-\mathrm{C} 8 \mathrm{~A}-\mathrm{C} 9 \mathrm{~A}$ & $114.4(12)$ \\
\hline $\mathrm{N} 2 \mathrm{~A}-\mathrm{C} 8 \mathrm{~A}-\mathrm{H} 8 \mathrm{AA}$ & 108.7 \\
\hline $\mathrm{C} 9 \mathrm{~A}-\mathrm{C} 8 \mathrm{~A}-\mathrm{H} 8 \mathrm{AA}$ & 108.7 \\
\hline $\mathrm{N} 2 \mathrm{~A}-\mathrm{C} 8 \mathrm{~A}-\mathrm{H} 8 \mathrm{AB}$ & 108.7 \\
\hline $\mathrm{C} 9 \mathrm{~A}-\mathrm{C} 8 \mathrm{~A}-\mathrm{H} 8 \mathrm{AB}$ & 108.7 \\
\hline $\mathrm{H} 8 \mathrm{AA}-\mathrm{C} 8 \mathrm{~A}-\mathrm{H} 8 \mathrm{AB}$ & 107.6 \\
\hline $\mathrm{C} 14 \mathrm{~A}-\mathrm{C} 9 \mathrm{~A}-\mathrm{C} 10 \mathrm{~A}$ & $123.7(18)$ \\
\hline $\mathrm{C} 14 \mathrm{~A}-\mathrm{C} 9 \mathrm{~A}-\mathrm{C} 8 \mathrm{~A}$ & $123.0(17)$ \\
\hline $\mathrm{C} 10 \mathrm{~A}-\mathrm{C} 9 \mathrm{~A}-\mathrm{C} 8 \mathrm{~A}$ & $113.2(16)$ \\
\hline $\mathrm{C} 11 \mathrm{~A}-\mathrm{C} 10 \mathrm{~A}-\mathrm{C} 9 \mathrm{~A}$ & $117.4(17)$ \\
\hline $\mathrm{C} 11 \mathrm{~A}-\mathrm{C} 10 \mathrm{~A}-\mathrm{H} 10 \mathrm{~A}$ & 121.3 \\
\hline $\mathrm{C} 9 \mathrm{~A}-\mathrm{C} 10 \mathrm{~A}-\mathrm{H} 10 \mathrm{~A}$ & 121.3 \\
\hline $\mathrm{C} 12 \mathrm{~A}-\mathrm{C} 11 \mathrm{~A}-\mathrm{C} 10 \mathrm{~A}$ & $119(2)$ \\
\hline $\mathrm{C} 12 \mathrm{~A}-\mathrm{C} 11 \mathrm{~A}-\mathrm{H} 11 \mathrm{~A}$ & 120.5 \\
\hline $\mathrm{C} 10 \mathrm{~A}-\mathrm{C} 11 \mathrm{~A}-\mathrm{H} 11 \mathrm{~A}$ & 120.5 \\
\hline $\mathrm{C} 11 \mathrm{~A}-\mathrm{C} 12 \mathrm{~A}-\mathrm{C} 13 \mathrm{~A}$ & $121(2)$ \\
\hline $\mathrm{C} 11 \mathrm{~A}-\mathrm{C} 12 \mathrm{~A}-\mathrm{H} 12 \mathrm{~A}$ & 119.6 \\
\hline $\mathrm{C} 13 \mathrm{~A}-\mathrm{C} 12 \mathrm{~A}-\mathrm{H} 12 \mathrm{~A}$ & 119.6 \\
\hline $\mathrm{I} 6-\mathrm{I} 7-\mathrm{I} 7 \mathrm{~A}-\mathrm{I} 6^{\mathrm{ii}}$ & $-2.0(11)$ \\
\hline $\mathrm{C} 6 \mathrm{~A}-\mathrm{N} 1 \mathrm{~A}-\mathrm{C} 2 \mathrm{~A}-\mathrm{C} 3 \mathrm{~A}$ & $0(2)$ \\
\hline $\mathrm{C} 1 \mathrm{~A}-\mathrm{N} 1 \mathrm{~A}-\mathrm{C} 2 \mathrm{~A}-\mathrm{C} 3 \mathrm{~A}$ & $177.8(15)$ \\
\hline $\mathrm{N} 1 \mathrm{~A}-\mathrm{C} 2 \mathrm{~A}-\mathrm{C} 3 \mathrm{~A}-\mathrm{C} 4 \mathrm{~A}$ & $-3(3)$ \\
\hline $\mathrm{C} 2 \mathrm{~A}-\mathrm{C} 3 \mathrm{~A}-\mathrm{C} 4 \mathrm{~A}-\mathrm{C} 5 \mathrm{~A}$ & $4(3)$ \\
\hline $\mathrm{C} 2 \mathrm{~A}-\mathrm{C} 3 \mathrm{~A}-\mathrm{C} 4 \mathrm{~A}-\mathrm{C} 7 \mathrm{~A}$ & $-174.6(15)$ \\
\hline $\mathrm{C} 3 \mathrm{~A}-\mathrm{C} 4 \mathrm{~A}-\mathrm{C} 5 \mathrm{~A}-\mathrm{C} 6 \mathrm{~A}$ & $-2(3)$ \\
\hline $\mathrm{C} 7 \mathrm{~A}-\mathrm{C} 4 \mathrm{~A}-\mathrm{C} 5 \mathrm{~A}-\mathrm{C} 6 \mathrm{~A}$ & $176.7(14)$ \\
\hline $\mathrm{C} 2 \mathrm{~A}-\mathrm{N} 1 \mathrm{~A}-\mathrm{C} 6 \mathrm{~A}-\mathrm{C} 5 \mathrm{~A}$ & $2(2)$ \\
\hline $\mathrm{C} 1 \mathrm{~A}-\mathrm{N} 1 \mathrm{~A}-\mathrm{C} 6 \mathrm{~A}-\mathrm{C} 5 \mathrm{~A}$ & $-175.8(14)$ \\
\hline $\mathrm{C} 4 \mathrm{~A}-\mathrm{C} 5 \mathrm{~A}-\mathrm{C} 6 \mathrm{~A}-\mathrm{N} 1 \mathrm{~A}$ & $-1(2)$ \\
\hline $\mathrm{C} 8 \mathrm{~A}-\mathrm{N} 2 \mathrm{~A}-\mathrm{C} 7 \mathrm{~A}-\mathrm{O} 1 \mathrm{~A}$ & $-8(2)$ \\
\hline $\mathrm{C} 8 \mathrm{~A}-\mathrm{N} 2 \mathrm{~A}-\mathrm{C} 7 \mathrm{~A}-\mathrm{C} 4 \mathrm{~A}$ & $166.8(14)$ \\
\hline $\mathrm{C} 5 \mathrm{~A}-\mathrm{C} 4 \mathrm{~A}-\mathrm{C} 7 \mathrm{~A}-\mathrm{O} 1 \mathrm{~A}$ & $-19(2)$ \\
\hline $\mathrm{C} 3 \mathrm{~A}-\mathrm{C} 4 \mathrm{~A}-\mathrm{C} 7 \mathrm{~A}-\mathrm{O} 1 \mathrm{~A}$ & $159.5(17)$ \\
\hline $\mathrm{C} 5 \mathrm{~A}-\mathrm{C} 4 \mathrm{~A}-\mathrm{C} 7 \mathrm{~A}-\mathrm{N} 2 \mathrm{~A}$ & $166.0(15)$ \\
\hline $\mathrm{C} 3 \mathrm{~A}-\mathrm{C} 4 \mathrm{~A}-\mathrm{C} 7 \mathrm{~A}-\mathrm{N} 2 \mathrm{~A}$ & $-16(2)$ \\
\hline $\mathrm{C} 7 \mathrm{~A}-\mathrm{N} 2 \mathrm{~A}-\mathrm{C} 8 \mathrm{~A}-\mathrm{C} 9 \mathrm{~A}$ & $-81(2)$ \\
\hline $\mathrm{N} 2 \mathrm{~A}-\mathrm{C} 8 \mathrm{~A}-\mathrm{C} 9 \mathrm{~A}-\mathrm{C} 14 \mathrm{~A}$ & $117.5(17)$ \\
\hline $\mathrm{N} 2 \mathrm{~A}-\mathrm{C} 8 \mathrm{~A}-\mathrm{C} 9 \mathrm{~A}-\mathrm{C} 10 \mathrm{~A}$ & $-61.6(18)$ \\
\hline $\mathrm{C} 14 \mathrm{~A}-\mathrm{C} 9 \mathrm{~A}-\mathrm{C} 10 \mathrm{~A}-\mathrm{C} 11 \mathrm{~A}$ & $0(3)$ \\
\hline $\mathrm{C} 8 \mathrm{~A}-\mathrm{C} 9 \mathrm{~A}-\mathrm{C} 10 \mathrm{~A}-\mathrm{C} 11 \mathrm{~A}$ & $179.4(14)$ \\
\hline $\mathrm{C} 9 \mathrm{~A}-\mathrm{C} 10 \mathrm{~A}-\mathrm{C} 11 \mathrm{~A}-\mathrm{C} 12 \mathrm{~A}$ & $2(3)$ \\
\hline $\mathrm{C} 10 \mathrm{~A}-\mathrm{C} 11 \mathrm{~A}-\mathrm{C} 12 \mathrm{~A}-\mathrm{C} 13 \mathrm{~A}$ & $-4(3)$ \\
\hline $\mathrm{C} 11 \mathrm{~A}-\mathrm{C} 12 \mathrm{~A}-\mathrm{C} 13 \mathrm{~A}-\mathrm{C} 14 \mathrm{~A}$ & $4(3)$ \\
\hline $\mathrm{C} 10 \mathrm{~A}-\mathrm{C} 9 \mathrm{~A}-\mathrm{C} 14 \mathrm{~A}-\mathrm{C} 13 \mathrm{~A}$ & $-1(3)$ \\
\hline
\end{tabular}

$\begin{array}{ll}\mathrm{C} 10 \mathrm{~B}-\mathrm{C} 9 \mathrm{~B}-\mathrm{C} 14 \mathrm{~B} & 118.1(15) \\ \mathrm{C} 10 \mathrm{~B}-\mathrm{C} 9 \mathrm{~B}-\mathrm{C} 8 \mathrm{~B} & 122.2(15) \\ \mathrm{C} 14 \mathrm{~B}-\mathrm{C} 9 \mathrm{~B}-\mathrm{C} 8 \mathrm{~B} & 119.6(15) \\ \mathrm{C} 11 \mathrm{~B}-\mathrm{C} 10 \mathrm{~B}-\mathrm{C} 9 \mathrm{~B} & 122.2(17) \\ \mathrm{C} 11 \mathrm{~B}-\mathrm{C} 10 \mathrm{~B}-\mathrm{H} 10 \mathrm{~B} & 118.9 \\ \mathrm{C} 9 \mathrm{~B}-\mathrm{C} 10 \mathrm{~B}-\mathrm{H} 10 \mathrm{~B} & 118.9 \\ \mathrm{C} 10 \mathrm{~B}-\mathrm{C} 11 \mathrm{~B}-\mathrm{C} 12 \mathrm{~B} & 120.2(18) \\ \mathrm{C} 10 \mathrm{~B}-\mathrm{C} 11 \mathrm{~B}-\mathrm{H} 11 \mathrm{~B} & 119.9 \\ \mathrm{C} 12 \mathrm{~B}-\mathrm{C} 11 \mathrm{~B}-\mathrm{H} 11 \mathrm{~B} & 119.9 \\ \mathrm{C} 13 \mathrm{~B}-\mathrm{C} 12 \mathrm{~B}-\mathrm{C} 11 \mathrm{~B} & 119.9(18) \\ \mathrm{C} 13 \mathrm{~B}-\mathrm{C} 12 \mathrm{~B}-\mathrm{H} 12 \mathrm{~B} & 120.1 \\ \mathrm{C} 11 \mathrm{~B}-\mathrm{C} 12 \mathrm{~B}-\mathrm{H} 12 \mathrm{~B} & 120.1 \\ \mathrm{C} 12 \mathrm{~B}-\mathrm{C} 13 \mathrm{~B}-\mathrm{C} 14 \mathrm{~B} & 119.9(17) \\ \mathrm{C} 12 \mathrm{~B}-\mathrm{C} 13 \mathrm{~B}-\mathrm{H} 13 \mathrm{~B} & 120.1 \\ \mathrm{C} 14 \mathrm{~B}-\mathrm{C} 13 \mathrm{~B}-\mathrm{H} 13 \mathrm{~B} & 120.1 \\ \text { C9B-C14B-C13B } & 119.6(16) \\ \text { C9B-C14B-H14B } & 120.2 \\ \text { C13B-C14B-H14B } & 120.2\end{array}$

$\begin{array}{ll}\mathrm{C} 6 \mathrm{~B}-\mathrm{N} 1 \mathrm{~B}-\mathrm{C} 2 \mathrm{~B}-\mathrm{C} 3 \mathrm{~B} & -7(3) \\ \mathrm{C} 1 \mathrm{~B}-\mathrm{N} 1 \mathrm{~B}-\mathrm{C} 2 \mathrm{~B}-\mathrm{C} 3 \mathrm{~B} & 176.0(14) \\ \mathrm{N} 1 \mathrm{~B}-\mathrm{C} 2 \mathrm{~B}-\mathrm{C} 3 \mathrm{~B}-\mathrm{C} 4 \mathrm{~B} & 6(3)\end{array}$

$\mathrm{N} 1 \mathrm{~B}-\mathrm{C} 2 \mathrm{~B}-\mathrm{C} 3 \mathrm{~B}-\mathrm{C} 4 \mathrm{~B}$

$\mathrm{C} 2 \mathrm{~B}-\mathrm{C} 3 \mathrm{~B}-\mathrm{C} 4 \mathrm{~B}-\mathrm{C} 5 \mathrm{~B} \quad-2(2)$

$\mathrm{C} 2 \mathrm{~B}-\mathrm{C} 3 \mathrm{~B}-\mathrm{C} 4 \mathrm{~B}-\mathrm{C} 7 \mathrm{~B} \quad 173.6(15)$

$\mathrm{C} 3 \mathrm{~B}-\mathrm{C} 4 \mathrm{~B}-\mathrm{C} 5 \mathrm{~B}-\mathrm{C} 6 \mathrm{~B} \quad-1(2)$

$\mathrm{C} 7 \mathrm{~B}-\mathrm{C} 4 \mathrm{~B}-\mathrm{C} 5 \mathrm{~B}-\mathrm{C} 6 \mathrm{~B} \quad-176.4(14)$

$\mathrm{C} 2 \mathrm{~B}-\mathrm{N} 1 \mathrm{~B}-\mathrm{C} 6 \mathrm{~B}-\mathrm{C} 5 \mathrm{~B} \quad 4$ (3)

$\mathrm{C} 1 \mathrm{~B}-\mathrm{N} 1 \mathrm{~B}-\mathrm{C} 6 \mathrm{~B}-\mathrm{C} 5 \mathrm{~B} \quad-178.7(15)$

$\mathrm{C} 4 \mathrm{~B}-\mathrm{C} 5 \mathrm{~B}-\mathrm{C} 6 \mathrm{~B}-\mathrm{N} 1 \mathrm{~B} \quad 0(3)$

$\mathrm{C} 8 \mathrm{~B}-\mathrm{N} 2 \mathrm{~B}-\mathrm{C} 7 \mathrm{~B}-\mathrm{O} 1 \mathrm{~B} \quad 3$ (2)

$\mathrm{C} 8 \mathrm{~B}-\mathrm{N} 2 \mathrm{~B}-\mathrm{C} 7 \mathrm{~B}-\mathrm{C} 4 \mathrm{~B} \quad-176.2(13)$

$\mathrm{C} 5 \mathrm{~B}-\mathrm{C} 4 \mathrm{~B}-\mathrm{C} 7 \mathrm{~B}-\mathrm{O} 1 \mathrm{~B} \quad 13(2)$

$\mathrm{C} 3 \mathrm{~B}-\mathrm{C} 4 \mathrm{~B}-\mathrm{C} 7 \mathrm{~B}-\mathrm{O} 1 \mathrm{~B} \quad-161.8(15)$

$\mathrm{C} 5 \mathrm{~B}-\mathrm{C} 4 \mathrm{~B}-\mathrm{C} 7 \mathrm{~B}-\mathrm{N} 2 \mathrm{~B} \quad-167.7(14)$

$\mathrm{C} 3 \mathrm{~B}-\mathrm{C} 4 \mathrm{~B}-\mathrm{C} 7 \mathrm{~B}-\mathrm{N} 2 \mathrm{~B} \quad 18(2)$

$\mathrm{C} 7 \mathrm{~B}-\mathrm{N} 2 \mathrm{~B}-\mathrm{C} 8 \mathrm{~B}-\mathrm{C} 9 \mathrm{~B} \quad 178.3$ (14)

$\mathrm{N} 2 \mathrm{~B}-\mathrm{C} 8 \mathrm{~B}-\mathrm{C} 9 \mathrm{~B}-\mathrm{C} 10 \mathrm{~B} \quad-53(2)$

$\mathrm{N} 2 \mathrm{~B}-\mathrm{C} 8 \mathrm{~B}-\mathrm{C} 9 \mathrm{~B}-\mathrm{C} 14 \mathrm{~B} \quad 124.6(15)$

$\mathrm{C} 14 \mathrm{~B}-\mathrm{C} 9 \mathrm{~B}-\mathrm{C} 10 \mathrm{~B}-\mathrm{C} 11 \mathrm{~B} \quad 3(2)$

$\mathrm{C} 8 \mathrm{~B}-\mathrm{C} 9 \mathrm{~B}-\mathrm{C} 10 \mathrm{~B}-\mathrm{C} 11 \mathrm{~B} \quad-179.5(15)$

$\mathrm{C} 9 \mathrm{~B}-\mathrm{C} 10 \mathrm{~B}-\mathrm{C} 11 \mathrm{~B}-\mathrm{C} 12 \mathrm{~B}-2(3)$

$\mathrm{C} 10 \mathrm{~B}-\mathrm{C} 11 \mathrm{~B}-\mathrm{C} 12 \mathrm{~B}-\mathrm{C} 13 \mathrm{~B}-2(3)$

$\mathrm{C} 11 \mathrm{~B}-\mathrm{C} 12 \mathrm{~B}-\mathrm{C} 13 \mathrm{~B}-\mathrm{C} 14 \mathrm{~B} \quad 4$ (2)

$\mathrm{C} 10 \mathrm{~B}-\mathrm{C} 9 \mathrm{~B}-\mathrm{C} 14 \mathrm{~B}-\mathrm{C} 13 \mathrm{~B} \quad 0(2)$

$\mathrm{C} 8 \mathrm{~B}-\mathrm{C} 9 \mathrm{~B}-\mathrm{C} 14 \mathrm{~B}-\mathrm{C} 13 \mathrm{~B} \quad-178.0(14)$ 
$\mathrm{C} 8 \mathrm{~A}-\mathrm{C} 9 \mathrm{~A}-\mathrm{C} 14 \mathrm{~A}-\mathrm{C} 13 \mathrm{~A} \quad 179.8(15)$

$\mathrm{C} 12 \mathrm{~B}-\mathrm{C} 13 \mathrm{~B}-\mathrm{C} 14 \mathrm{~B}-\mathrm{C} 9 \mathrm{~B}-3(2)$

$\mathrm{C} 12 \mathrm{~A}-\mathrm{C} 13 \mathrm{~A}-\mathrm{C} 14 \mathrm{~A}-\mathrm{C} 9 \mathrm{~A}-1(3)$

Symmetry codes: (i) $-x,-y+2,-z+1$; (ii) $-x-1,-y+1,-z+1$.

Hydrogen-bond geometry $\left(A,{ }^{\circ}\right)$

\begin{tabular}{lllll}
\hline$D-\mathrm{H} \cdots A$ & $D-\mathrm{H}$ & $\mathrm{H} \cdots A$ & $D \cdots A$ & $D-\mathrm{H} \cdots A$ \\
\hline $\mathrm{N} 2 A-\mathrm{H} 2 A \cdots \mathrm{O} 1 B$ & 0.86 & 2.02 & $2.846(14)$ & 160 \\
$\mathrm{C} 3 A-\mathrm{H} 3 A \cdots \mathrm{O} 1 B$ & 0.93 & 2.53 & $3.381(18)$ & 152 \\
$\mathrm{C} 2 A-\mathrm{H} 2 A A \cdots \mathrm{I} 3$ & 0.93 & 3.08 & $3.998(17)$ & 169 \\
$\mathrm{C} 1 A-\mathrm{H} 1 A C \cdots \mathrm{C} 12 A^{\mathrm{iii}}$ & 0.96 & 2.72 & $3.62(2)$ & 158 \\
$\mathrm{C} 1 A-\mathrm{H} 1 A A \cdots \mathrm{I} 7^{\mathrm{iii}}$ & 0.96 & 3.09 & $3.966(19)$ & 153 \\
$\mathrm{~N} 2 B-\mathrm{H} 2 B \cdots \mathrm{O} 1 A^{\mathrm{iv}}$ & 0.86 & 2.13 & $2.986(14)$ & 176 \\
$\mathrm{C} 3 B-\mathrm{H} 3 B \cdots \mathrm{O} 1 A^{\mathrm{iv}}$ & 0.93 & 2.21 & $3.060(17)$ & 151 \\
$\mathrm{C} 2 B-\mathrm{H} 2 B A \cdots \mathrm{C} 12 A^{\mathrm{v}}$ & 0.93 & 2.85 & $3.72(2)$ & 156 \\
$\mathrm{C} 1 B-\mathrm{H} 1 B B \cdots \mathrm{I} 7^{\mathrm{vi}}$ & 0.96 & 3.07 & $3.819(18)$ & 136 \\
$\mathrm{C} 6 B-\mathrm{H} 6 B \cdots \mathrm{I} 4^{\mathrm{vii}}$ & 0.93 & 3.12 & $4.019(17)$ & 164
\end{tabular}

Symmetry codes: (iii) $x,-y+3 / 2, z-1 / 2$; (iv) $x+1, y, z$; (v) $-x+1,-y+1,-z+1$; (vi) $-x,-y+1,-z+1$; (vii) $-x, y-1 / 2,-z+1 / 2$. 